\title{
Philosophiques
}

\section{Yvon Gauthier, De la logique interne, Paris, Librairie philosophique J. Vrin, collection Mathesis, 1991, 140 pages.}

\section{Mathieu Marion}

Volume 22, numéro 2, automne 1995

URI : https://id.erudit.org/iderudit/027335ar

DOI : https://doi.org/10.7202/027335ar

Aller au sommaire du numéro

Éditeur(s)

Société de philosophie du Québec

ISSN

0316-2923 (imprimé)

1492-1391 (numérique)

Découvrir la revue

Citer cet article

Marion, M. (1995). Yvon Gauthier, De la logique interne, Paris, Librairie philosophique J. Vrin, collection Mathesis, 1991, 140 pages. Philosophiques,

22(2), 301-336. https://doi.org/10.7202/027335ar d'utilisation que vous pouvez consulter en ligne.

https://apropos.erudit.org/fr/usagers/politique-dutilisation/ 


\section{E I U U D E}

Yvon Gauthier, De la logique interne, Paris,

Librairie philosophique J. Vrin, collection

Mathesis, 1991, 140 pages

par Mathieu Marion"

La demière décennie a vu un essor considérable de la recherche sur les fragments de l'arithmétique de Peano. Parallèlement, la mathématique non standard a continué à croître en popularité, surtout après l' introduction du nouveau langage de la théorie inteme des ensembles créé par Edward Nelson'. Pour des raisons d'espace, je me contenterai de discuter l'ouvrage d'Yvon Gauthier, De la logique interne, en relation avec ces deux domaines florissants. Je compte aussi m'attarder sur les sections dédiées à Frege, Weyl et Wittgenstein. Je ne prétends pas faire justice à la richesse de l'ouvrage de Gauthier, dont $\mathrm{j}$ 'ai dû passer sous silence bien des aspects. Le mieux que je puisse espérer ici, c'est d'être arrivé à poser quelques bonnes questions. Le lecteur me pardonnera mon ton pédagogique qui allonge le texte par endroit: la littérature de langue française dans ce domaine est quasi-inexistante.

* N.D.L.R. Philosophiques reprend ici cette étude critique précédemment publiée dans nos pages et qui, malheureusement, avait été rendue incompréhensible à la suite d'erreurs techniques indépendantes de notre volonté. Le comité de rédaction présente toutes ses excuses à $M$. Mathieu Marion.

1. E. Nelson, «Intemal Set Theory: A New Approach to Nonstandard Analysis ", Bulletin of the American Mathematical Society 83, 1977, p. 1165-1198; traduction française dans H. Barreau \& J. Harthong (dir.), La mathématique non standard, Paris, Éditions du C.N.R.S., 1989, p. 355-399. On trouvera une introduction à la littérature sur les fragments de l'arithmétique, aussi appelés systèmes faibles, dans le "manifeste " d'Angus Macintyre, "The Strength of Weak Systems", dans P. Weingartner \& G. Schurz, (dir.), Logic, Philosophy of Science and Epistemology. Proceedings of the 11th International Wittgenstein Symposium, Vienne, Hölder-Pichler-Tempsky, 1987, p. 43-59. Pour juger de la fécondité de l'analyse non standard, le lecteur consultera N. Cutland (dir.), Nonstandard Analysis and its Applications, Cambridge, Cambridge University Press, 1988. 


\section{De la logique interne à l'arithmétique de Fermat}

Dès les premières pages, Gauthier nous invite à « imaginer une hiérarchie de logiques internes en commençant par la plus simple ou la plus pauvre » (p. 8), et il propose sa logique de la négation locale comme étant celle qui «doit servir comme un fondement de toutes les logiques » (p. 15). Pour cela, Gauthier propose de s'en tenir à « l'interprétation la plus stricte possible des constantes logiques (connecteurs et quantificateurs) et des règles d'inférence ou méthodes de preuve » (p. 23). Ce n'est cependant que dans la troisième partie de l'ouvrage que Gauthier esquisse son système de logique, qui se distingue de la logique intuitionniste en ce que non seulement $\neg \neg a \rightarrow a$ est rejeté mais aussi $a \rightarrow \neg \neg a$, puisque la négation est représentée de manière topologique par la différence arithmétique entre un domaine et son extérieur et qu'il n'y a pas de symétrie entre une assertion dans un domaine quelconque $D$ et sa double négation : $\mathrm{D}(a) \neq \mathrm{D}(\neg \neg a)$ (p. 98).

Afin de rendre sa logique de la négation locale "pauvre » mais arithmétiquement «forte », Gauthier lui donne une représentation arithmétique : la conjonction correspond à la multiplication et la disjonction à l'addition, tandis que l'implication est représentée - c'est là un aspect original - comme produit de Cauchy de séries de puissances :

$$
\sum_{0}^{\infty} c_{n} x^{n}=\sum_{0}^{\infty} a_{n} x^{n} \times \sum_{0}^{\infty} b_{n} x^{n}
$$

où $c_{n}=a_{O} b_{n}+a_{1} b_{n-1}+\ldots a_{n} b_{O}$, ce qui lui donne un contenu arithmétique (p. $101 \&$ 123). Le quantificateur existentiel est interprété de manière intuitionniste comme une instance numérique (ou une somme d'instances numériques) et le quantificateur universel est interprété comme un produit d'instances numériques. Gauthier n'utilise cependant ces quantificateurs que sur des domaines finis. En effet, il ne veut pas de ceux-ci pour rendre compte des suites infinies ${ }^{2}$, puisqu'il voit en eux le germe de la « totification », c'està-dire de l'ensemblage des nombres naturels qu'opère la théorie des ensembles : « le quantificateur universel a l'effet de transformer une suite effinie en ensemble infini, c'est-à-dire en totalité infinie complétée, ce qui est illégitime

2. Plus exactement, Gauthier veut rendre compte des suites infiniment processives de Brouwer. Je ne sais pas jusqu'où Gauthier veut ou peut aller dans ce sens, puisque l'introduction de variables $\phi$ pour des suites de choix est habituellement accompagnée de l'introduction de fonctionnelles $\tau(\phi)$, avec $\mathrm{P}(\phi, \tau(\phi)$, si $\exists x \mathrm{P}(\phi, x)$ a été prouvé, ce qui fait perdre au système son caractère purement arithmétique. 
à nos yeux » (p. 75). Gauthier introduit donc le quantificateur effini, qui s'écrit $\Xi x$ et « doit se lire "pour tous les $x$ effiniment ", c'est-à-dire pour tous les membres d'une suite processive potentiellement infinie" (p. 98). Cette distinction de Gauthier rappelle les mots de Wittgenstein :

Ce n'est nullement un seul et même concept qui serait déterminé plus précisément par l'adjonction de « fini » ou « infini ».

Russell a favorisé davantage encore cette erreur en créant un symbolisme qui représente les deux types de classes exactement de la même façon.

$[\ldots]$

Un symbolisme approprié doit représenter une classe infinie tout autrement qu'une classe finie ${ }^{3}$.

Il faut s'arrêter ici un instant et se demander qu'elle est la différence entre le quantificateur effini de Gauthier et le quantificateur universel des intuitionnistes. Voici comment Michael Dummett décrit la notion intuitionniste de procès infini :

[...] la thèse selon laquelle il n'y a pas d'infini complété signifie simplement que saisir une structure infinie, c'est saisir un procès qui la génère, et que réfèrer à une telle structure, c'est réferer à ce procès, et reconnaître la structure comme infinie, c'est reconnaître que le procès n'a pas de fin ${ }^{4}$.

Cette lecture informelle ne differe que de peu de celle de Gauthier. La différence est cependant cruciale puisque, comme tous les intuitionnistes depuis Brouwer, Dummett admet les ordinaux transfinis $\alpha<\varepsilon_{0}$ tandis que pour Gauthier « il n'y a pas de $\omega »(\text { p. } 121)^{5}$. C'est qu'il rejette tout simplement l'interprétation ensembliste de $\omega$ comme procès infini achevé et que pour lui les ordinaux $\alpha<\varepsilon_{0}$ n'ont aucune signification.

En cela, Gauthier a maintenant un allié de taille : Edward Nelson, pour qui « la foi en $\omega$ c'est de l'idolâtrie ${ }^{6}$ ». Je n'ai pas l'intention de critiquer les

3. L. Wittgenstein, Wittgenstein et le Cercle de Vienne, traduction de G. Granel, Mauzevin, Trans-Europ-Repress, 1991, p. 210. (Traduction légèrement modifiée.)

4. M. A. E. Dummett, Elements of Intuitionism, Oxford, Oxford University Press, 1977, p. 56.

5. Pour ce qui est de Dummett, je prends comme exemple son tout récent aveu : « on se sent tout à fait à l'aise avec la notion de nombres cardinaux transfinis », ("What is Mathematics About? », dans M. A. E. Dummett, The Seas of Language, Oxford, Oxford University Press, 1993, p. 440 ; ce texte devrait aussi paraître dans A. George (dir.) Mathematics and Mind, Oxford, Oxford University Press).

6. E. Nelson, Predicative Arithmetic, Princeton, Princeton University Press, 1986, p. 80 . 
travaux de Gauthier d'un point de vue ensembliste. J'aimerais cependant observer le décalage entre ses ambitions finitistes et la force de son formalisme. À mon avis un affaiblissement de l'arithmétique de Peano, qui consiste à restreindre l'induction aux formules bornées dites $\Delta_{0}$, permet d'obtenir un système à justification finitiste plus faible encore que celui de Gauthier. Le "programme de Hilbert modifié " mis de l'avant par Edward Nelson dans le dernier chapitre de son livre Predicative Arithmetic, est basé sur cette arithmétique bomée. Je reviendrai sur cette question dans la prochaine section. Il faut tout d'abord se pencher sur l'aspect central du livre de Gauthier, la « descente infinie ». En effet, pour arriver à l'arithmétique, il faut ajouter à la logique un certain nombre d'axiomes non-logiques, pour $0, S,+, \cdot<$ et il faut ajouter un principe d'induction. L'arithmétique de Peano se caractérise par le schéma d'induction $I(\phi)$ :

$$
[\phi(0) \wedge \forall x(\phi(x) \rightarrow \phi(S x))] \rightarrow \forall x(\phi(x))
$$

Gauthier veut cependant remplacer celui-ci par un schéma dérivé de la méthode dite de "descente infinie » que l'on doit à Pierre de Fermat. Selon le théoricien des nombres Harold Edwards, cette méthode repose sur le principe suivant :

Si la supposition qu'un entier positif possède un ensemble donné de propriétés implique l'existence d'un plus petit entier positif possèdant le même ensemble de propriétés, alors aucun entier positif ne possède cet ensemble de propriétés ${ }^{7}$.

La méthode de descente fonctionne donc par reductio ad absurdum. En effet, si la supposition qu'un entier positif $x$ possède la propriété $\phi$ implique l'existence un $y<x$ possèdant aussi la propriété $\phi$, alors cette supposition implique que l'on se trouve devant une suite décroissante infinie d'entiers positifs ; mais cette descente infinie est impossible, puisqu'on doit nécessairement aboutir à zéro. Donc, par reductio, aucun entier positif ne peut posséder la propriété $\phi$.

Ce procédé semble de prime abord légitime du point de vue constructiviste, puisqu' on reste dans le fini en «descendant » jusqu'à zéro. Cependant, si on se place du point de vue classique, on peut dériver le principe d'induction complète :

$$
\forall x[\forall y<x \rightarrow \phi(y)] \rightarrow \forall x \phi(x)
$$

à partir du schéma $\mathrm{I}(\phi)$. L'induction complète sert à son tour à obtenir le prin-

7. H. M. Edwards, Fermat's Last Theorem, Berlin, Springer, 1977, p. 9. 
cipe du plus petit nombre en quelques étapes, lorsqu'on remplace $\phi$ par $\neg \phi$ :

$$
\exists x \phi(x) \rightarrow \exists x\left(\phi(x) \wedge(\forall y<x \rightarrow \neg \phi(y))^{8}\right.
$$

et il est coutumier de dériver le principe de la descente infinie de Fermat à partir de celui-ci. Je donne la formalisation suivante pour la forme classique du principe :

$$
\text { (DI) } \forall x[\phi(x) \rightarrow(\exists y<x \rightarrow \phi(y))]
$$

Encore une fois, ce principe fonctionne par reductio ad absurdum. Supposons qu'on veuille prouver $\forall x \phi(x)$. On part donc de $\neg \forall x \phi(x)$, ce qui est équivalent, classiquement, à $\exists x \neg \phi(x)$. Par DI, il y a donc un $y<x$ tel que $\neg \phi(y)$. En répétant l'utilisation de DI indéfiniment, on obtient une suite infinie de nombres de plus en plus petits possédant la propriété $\phi$, ce qui ne peut pas être le cas. Donc $\forall x \phi(x)$. On peut formaliser ce raisonnement de la façon suivante :

$$
\forall x[\phi(x) \rightarrow(\exists y<x \rightarrow \phi(y))] \rightarrow \exists y \forall z<y \phi(z)
$$

Cette descente infinie correspond à l'induction complète et n'a donc aucun statut particulier. Elle n'a pas le caractère plus spécifiquement finitiste que lui prête Gauthier. Malgré que même un Nelson considère l'usage indirect du principe du plus petit nombre comme équivalent à une preuve par la descente infinie ${ }^{9}$, Gauthier s'oppose à ce raisonnement. C'est ici une question d'ordre conceptuel : Gauthier est sensible à l'aspect finitiste de la descente infinie qu'il ne veut pas associer à l'induction de Peano, qui n'est en fin de compte qu'une notion ensembliste.

Puisque le quantificateur universel qui apparaît dans DI est sans bome, il doit être remplacé, selon Gauthier, par le quantificateur effini. Il récrit donc le raisonnement comme suit :

$$
\Xi x[(\phi(x) \rightarrow \exists y<x \phi(y)) \rightarrow \exists y \Xi z<y \phi(z)] \rightarrow \Xi x \neg \phi(x)
$$

ce qui signifie

[...] que si l'existence d'une propriété pour un nombre donné $n$ implique l'existence de cette propriété pour un nombre plus petit quelconque, alors la propriété est possédée par la suite effinie descendante des nombres naturels, ce qui est impossible, donc la propriété est fausse pour tous les nombres naturels « effiniment ». (p. 77)

En insistant sur l'aspect finitiste, Gauthier dit vouloir revenir par-delà

8. J. R. Schoenfield, Mathematical Logic, Reading Mass., Addison-Wesley, 1967, p. 205.

9. E. Nelson, Predicative Arithmetic, op. cit., p. 29. 
l'approche ensembliste de Peano à la pratique mathématique en « théorie des nombres telles qu'on l'a pratiquée depuis Fermat - et avant lui, Diophante, Euclide, les Babyloniens — jusqu'à maintenant » (p. 23). Il faut en effet prendre un recul historique et surtout ne pas négliger que la découverte des quantificateurs et la formalisation du principe d'induction qui s'en est suivi datent de la deuxième moitié du XIX ${ }^{e}$ siècle. II se peut que ceux-ci ne correspondent pas à la pratique antérieure en théorie des nombres. Cependant, du point de vue classique (à la suite de Cantor et Peano) pour lequel la descente infinie est équivalente à l'induction transfinie, il n'y a pas de différence essentielle entre la pratique mathématique qui précède et celle qui suit l'introduction de l'induction transfinie. Ce point de vue est illustré par Georg Kreisel :

Lorsque l'induction transfinie fut formulée pour la première fois au siècle dernier, la Méthode était déjà connue pour l'essentiel depuis Fermat. En effet, on n'avait utilisé jusqu'à cette époque aucun prédicat quantifié, mais bien plutôt seulement des équations ou des inégalités [...] Par exemple, on prouverait aujourd'hui

$$
(p+1)^{3}+(q+1)^{3} \neq(n+1)^{3}
$$

en bref,

$$
F_{3}(p, q, n)
$$

au moyen de l'induction ordinaire

$$
\forall \mathbf{p} \forall \mathbf{q} F_{3}(\mathbf{p}, \mathbf{q}, \mathbf{n})
$$

Avant que l'on se soit habitué aux symboles logiques, le triplet (n, p, q) était ordonné lexicographiquement et la Méthode de descente employée. Il serait concevable qu'on produise une preuve de la conjecture de Fermat

$$
(\mathrm{p}+1)^{\mathrm{r}+3}+(\mathrm{q}+1)^{\mathrm{r}+3} \neq(\mathrm{n}+1)^{\mathrm{r}+3}
$$

à l'aide d'un ordre approprié du quadruplet $(p, q, r, n)$ - avec quelque chose comme $\boldsymbol{\varepsilon}_{0}$ comme nombre ordinal ${ }^{10}$ !

Le pari de Gauthier consiste donc à montrer que la descente infinie entendue au sens constructiviste differe de celle entendue au sens classique et qu'elle correspond bien au principe dont les théoriciens du nombre firent et font encore usage. Du même coup, la descente infinie comprise comme l'équivalent de l'induction complète ne deviendrait qu'une invention des logiciens, plus précisément une perversion qui a son origine dans les travaux de Gentzen.

10. G. Kreisel, «Wie die Beweistheorie zu ihren Ordinalzahlen kam und kommt », Jahresbericht der deutschen Mathematiker-Vereinigung 78, 1976, p. 186. 
Il faut noter à l'appui de Gauthier que son point de vue respecte mieux l'esprit de Fermat. Il n'est pas difficile de vérifier que Fermat utilisait avec prédilection la descente infinie, puisqu'on ne lui doit qu'une seule preuve ${ }^{11}$, celle du théorème selon lequel l'équation $x^{4}+y^{4}=z^{2}$ n'a pas de solution dans les nombres entiers pour $x, y, z \neq 0$ et $z>0$. (Celle-ci est reproduite dans l'appendice I (p. 109-110).) Voilà le hic : la descente infinie est une méthode tout à fait appropriée à la théorie des formes quadratiques. Ces quelques lignes d'André Weil le montre bien :

Les propos de Fermat indiquent qu'il était en possession de la théorie des formes quadratiques $X^{2}+n Y^{2}$ pour $n=1,2,3$ utilisant des preuves par « descente infinie ». Il ne décrivit pas ces preuves; mais en fin de compte Euler developpa cette théorie en utilisant aussi la descente infinie, de telle sorte qu'on peut présumer que les preuves de Fermat ne diffèrent pas vraiment de celles d'Euler. D'où vient le succès de la descente infinie dans ces cas ? L'historien qui sait que les corps quadratiques correspondants possèdent un algorithme euclidien l'explique aisément ; ce dernier, une fois traduit dans le langage et les notations de Fermat et Euler redonne précisément leur preuves par descente infinie, $[\ldots]^{12}$.

Weil a souligné plusieurs fois la parenté de l'algorithme d'Euclide avec la descente infinie de Fermat. Selon noi, c'est dans celle-ci que réside la constructivité de l'arithmétique avec descente infinie de Gauthier. On voit bien d'autre part que la notion logique moderne de «descente», équivalente à l'induction complète, a perdu ce caractère constructif.

Il faut aussi noter que l'introduction de l'induction dans l'arithmétique de Peano causa quelques remous. Déjà, à l'époque où il querellait avec les "péaniens " par excellence que sont Russell et Couturat, Poincaré avait fait la remarque que la descente infinie ne correspond pas à l'induction complète ${ }^{13}$.

11. Ce qui ne veut pas dire qu'il n'a pas ohtenu d'autres résultats, seulement qu'il ne divulguait pas ses preuves, ce qui était pratique courante à l'époque : lorsqu'un mathématicien obtenait une preuve, il mettait au défi les mathématiciens étrangers de la retrouver. Le cas de l'équation de Pell, l'équation $N x^{2}+1=y^{2}$ à résoudre pour $x$ et $y$ nombres entiers et $N$ un nombre entier donné non carré, est un éxemple parfait : Fermat disait avoir obtenu une preuve générale par « descente "; son défi fut relevé par les mathématiciens anglais, dont Pell (d'où le nom donné à l'équation aujourd'hui). Euler retrouva, quelques années plus tard, la preuve de Fermat.

12. A. Weil, "History of Mathematics: Why and How ", dans A. Weil, Euvres scientifiques. Collected papers, vol. 3, Berlin, Springer, 1979, p. 439.

13. Henri Poincaré, "Les mathématiques et la logique ", Revue de métaphysique et de morale 13, 1906, p. 303. 
J'ai aussi trouvé dans les pages de la Revue de métaphysique et de morale un débat portant ces principes entre deux italiens, Giovanni Vacca et l'élève de Peano, Alessandro Padoa ${ }^{14}$. Vacca donne deux versions de la descente infinie, une version négative qui correspond à celle mentionnée plus haut et une version positive :

Si le nombre zéro jouit d'une certaine propriété $\mathrm{R}$, et si l'on sait, par un procédé général, réduire la démonstration de la propriété $\mathrm{R}$, relative au nombre $n$, autre que zéro, à la démonstration de la même propriété $\mathrm{R}$ pour un nombre moindre que $n$, alors tout nombre jouit de la propriété $\mathrm{R}^{15}$.

Pour Vacca, la descente infinie ne peut pas se réduire à l'induction mathématique $^{16}$. Le procédé était jugé finitiste, puisqu'on reste dans le fini en "descendant " jusqu'à zéro. Comme Vacca le disait lui-même pour sa seconde version du principe :

La vérité de ce principe résulte de ce qu'on pourra, l'appliquant un nombre fini de fois, arriver au nombre zéro pour lequel la propriété $\mathrm{R}$ est vérifiée ${ }^{17}$.

Mais ce sont là des cas isolés et la méthode de descente a perdu beaucoup d'intérêt chez les logiciens au XX siècle, malgré qu'elle soit restée fort utile en mathématique. Elle a joué en particulier un rôle prépondérant dans la preuve du théorème de Mordell qui dit qu' une courbe algébrique de genre 1 sur un corps de nombre (i.e. une courbe elliptique) a au plus un nombre fini de points rationnels sur celui-ci, dans les travaux subséquents de Weil ${ }^{18}$ et plus récemment dans la preuve de la conjecture de Mordell sur les courbes de genre au moins 2 par Gerd Faltings ${ }^{19}$. Notons au passage que c'est un «programme d'étude» proposé par Poincaré dans l'article «Sur l'arithmétique des courbes algébriques ${ }^{20} "$ qui est à l'origine des travaux de Mordell

14. Giovanni Vacca, «Sur le principe d'induction mathématique », Revue de métaphysique et de morale 19, 1911, p. 29-33, "Sur la logique de la théorie des nombres », Revue de métaphysique et de morale 19, 1911, p. 252-257, et A. Padoa, "Sur le principe d'induction mathématique », Revue de métaphysique et de morale 19,1911 , p. 246-249.

15. G. Vacca, «Sur le principe d'induction mathématique», op. cit., p. 32.

16. Ibid.

17. Ibid

18. A. Weil, «L'arithmétique sur les courbes algébriques », dans A. Weil, Euvres scientifiques. Collected papers, op. cit, vol. 1, p. 11-45.

19. G. Faltings, « Endlicheitssätze für abelsche Varietäten über Zahlkörpern », Inventiones Mathematicae 73, 1983, p. 349-366.

20. H. Poincaré, «Sur l'arithmétique des courbes algébriques», dans Euvres de 
et Weil. Poincaré lui-même utilisait la méthode de descente dans ce texte ${ }^{21}$, comme l'avait jadis judicieusement remarqué Weil ${ }^{2}$.

\section{L'arithmétique de Fermat contre l'arithmétique bornée}

Les fragments de l'arithmétique de Peano sont obtenus en restreignant le schème d'induction aux différentes classes $\mathbf{C}$ de formules de la hiérarchie arithmétique, telles que $E_{0}, \exists_{n+1}, \forall_{n+1}, E_{n+1}, U_{n+1}, \Delta_{0}, \Sigma_{n+1}, \Pi_{n+1}$. Pour $\phi \in \mathbf{C}$, et $\mathbf{C}$ une de ces classes, la théorie axiomatisée par $\mathrm{P}^{-}$et le schème d'induction

$$
\forall y((\phi(y, 0)) \wedge \forall x(\phi(x, y) \rightarrow \phi S x, y))) \rightarrow \forall x(\phi(x, y))
$$

est dénotée par IC. La force relative de ces systèmes est :

$$
\mathrm{IE}_{0} \subset \mathrm{IE}_{1} \subseteq \mathrm{IE}_{2} \subseteq \ldots \subseteq \mathrm{I} \Delta_{0} \subset \mathrm{I} \Sigma_{1} \subset \mathrm{I} \Sigma_{2} \subset \ldots \subset \mathrm{PA}
$$

( $\mathrm{IE}_{0}$ dénote ici le système de l'induction ouverte et le symbole $\subseteq$ dénote une inclusion dont on ne sait pas encore si elle est stricte ou non ${ }^{23}$.) Rien n'interdit une comparaison, ne serait-ce qu' informelle, entre l'arithmétique de Fermat et ces sous-systèmes. Tout d'abord, il faut préciser que Gauthier réclame pour son arithmétique de Fermat le statut cadre logique minimal, suffisamment fort pour pouvoir accomoder les preuves arithmétiques en théorie des nombres ${ }^{24}$. Ce réquisit élimine en partant certains sous-systèmes, tels que $\mathrm{IE}_{0}$ : Shepherdson a démontré, il y a déjà longtemps ${ }^{25}$, qu'on ne peut même pas

Henri Poincaré, Paris, Gauthier-Villars, 1950, vol. 5, p. 483-548.

21. Ibid., section VIII.

22. A. Weil, " L'arithmétique sur les courhes algébriques", op. cit., p. 12-13, "Arithmetic on Algebraic Varieties », dans A. Weil, Euvres scientifiques. Collected papers, op. cit, vol. 1, p. 116-117.

23. Pour plus de détails, le lecteur pourra consulter R. Kaye, Models of Peano Arithmetic, Oxford, Oxford University Press, 1991, ou P. Hàjek \& P. Pudlàk, Metamathematics of First-Order Arithmetic, Berlin, Springer, 1993.

24. Soit-dit en passant, il n'est pas évident que l'exemple préféré de Gauthier, la preuve « élémentaire " par Selberg et Erdös du théorème sur la distribution des nombres premiers puisse être accomodé dans son système! Il en est de même pour $\mathrm{I} \Delta_{0}$. Sur ce sujet, je réfère à l'étude de Paola D'Aquino sur le théorème de Tchebychef, "Local Behaviour of the Chebyshev Theorem in Models of I $\Delta_{0}$ ", Joumal of Symbolic Logic 57, 1992, p. 12-27.

25. J. C. Shepherdson, « Non-Standard Models for Fragments of Number Theory », dans . . W. Addison, L. Henkin \& A. Tarski (dir.), The Theory of Models. Proceedings of the 1963 International Symposium at Berkeley, Amsterdam, NorthHolland, 1965, p. 342-358. 
prouver dans $\mathrm{IE}_{0}$ un problème diophantien aussi simple que l'irrationalité de $\sqrt{2}$, c'est-à-dire

$$
\forall x, y(x+1)^{2} \neq 2(y+1)^{2} .
$$

Il y a cependant d'autre candidats, en particulier le système I $\Delta_{0}$ et son extension $I \Delta_{0}+\Omega_{1}$. Ceux-ci n'ont certes pas la même signification arithmétique que $\mathrm{IE}_{0}$ ou que le fragment algébrique de Prezburger ${ }^{26}$, mais ils ont beaucoup d'équivalents qui ne manquent pas d'intérêt. Sam Buss a démontré dans Bounded Arithmetic ${ }^{27}$ l'équivalence des fragments appelés $\mathrm{U}_{\mathrm{i}} \mathrm{T}_{2}^{i}$ et $\mathrm{U}_{\mathrm{i}} \mathrm{S}_{2}^{\mathrm{i}}$ de l'arithmétique bornée $S_{2}^{1}$ avec $I \Delta_{0}+\Omega_{1}$. On peut aussi mentionner ici le système PV (polynomially-verifiable) des torontois Stephen Cook et Alasdair Urquhart $^{28}$, dont le slogan est : « la preuve constructive est à l'algorithme ce que la preuve constructive faisable est à l'algorithme [computable] en temps polynomial ${ }^{29}{ }_{\gg}$. Buss a aussi montré que $S_{2}^{1}(\mathrm{PV})$, c'est-à-dire $S_{2}^{1}$ augmenté de la notation et des théorèmes de $\mathrm{PV}$, est une extension conservative de $\mathrm{PV}^{30}$. Les travaux de Nelson ${ }^{31}$, où les rapports entre les extensions de l'arithmétique $Q$ de Robinson et $I \Delta_{0}+\Omega_{1}$ sont étudiés, ont mis en relief l'intérêt fondationel de tous ces systèmes. Selon moi, il n'y pas de système plus faible que ceux-ci qui possèderait encore un tel intérêt, ce qui implique que l'arithmétique de Fermat n'est pas le cadre minimal que recherche Gauthier. Je ne vois pas

26. Voir les explications de Macintyre dans « The Strength of Weak Systems», op. cit., p. 49.

27. S. R. Buss, Bounded Arithmetic, Naples, Bibliopolis, 1986.

28. S. A. Cook \& A. Urquhart, « Functional Interpretations of Feasibly Constructive Arithmetic », Annals of Pure and Applied Logic 63, 1993, p. 103-201.

29. Ibid., p. 104.

30. Cf. S. R. Buss, Bounded Arithmetic, op. cit., p. 109. Il faut aussi mentionner le récent article de Jean-Yves Girard, Phil Scott et Andrej Scedrov sur la logique linéaire bornée, «Bounded Linear Logic: A Modular Approach to PolynomialTime Computability » (Theoretical Computer Science 97, 1992, p. 1-66). La logique linéaire est une de ces « éco-logiques " qui fait attention à ses ressources. On y distingue les ressources qui sont limitées de celles qui ne le sont pas. Les symboles ! et ? sont utilisés pour désigner les ressources illimitées : ! A signifie que A est mis en mémoire et peut-être utilisé un nombre illimité de fois. Dans la logique linéaire bornée, A pourra toujours être réutilisé, mais seulement un nombre limité de fois, déterminé à l'avance ; ' $x$ A signifie donc que A est mis en mémoire et peut être utilisé $x$ fois. Le système de logique linéaire bornée encode toutes (et seulement) les fonctions computables en temps polynomial. On rejoint ici $\mathrm{I} \Delta_{0}+\Omega_{1}$

31. E. Nelson, Predicative Arithmetic, op. cit., et Radically Elementary Probability Theory, Princeton, Princeton University Press, 1987. 
comment Gauthier peut affirmer (p. 69, note 1) que son arithmétique de Fermat avec sa descente infinie est «plus restreinte » que les systèmes dérivés de $I \Delta_{0}$ étudiés et utilisés par Buss, Nelson et les autres.

Pour les besoins de la discussion, je rappelle brièvement que, pour le langage $\mathrm{L}=\{0, \mathrm{~S},+, \cdot, \leq\}$ de la logique du premier ordre de l'arithmétique, les quantificateurs bornés sont, en abrégé, $(\forall x \leq t)$ et $(\exists x \leq t)$, pour $t$ un terme quelconque de L. Une formule $\phi(x, y)$ est $\Delta_{0}$ si tous ses quantificateurs sont bornés et le système I $\Delta_{0}$ est axiomatisé par le schème :

$$
\forall x, z((\phi(x, 0)) \wedge \forall y \leq z(\phi(x, y) \rightarrow \phi(x, S y))) \rightarrow \forall y \leq z(\phi(x, y))
$$

ainsi que par des axiomes, sous forme équationelle, définissant les propriétés arithmétiques de $0, S,+,^{\cdot}$ et $\leq$. On sait depuis les travaux de Rohit Parikh ${ }^{32}$, que si $\phi(x, y)$ est $\Delta_{0}$ et

$$
\mathrm{I} \Delta_{0} \vdash \forall x \exists y \phi(x, y),
$$

alors

$$
\mathrm{I} \Delta_{0} \vdash \forall x \exists y \leq\left(\max \left(2+x_{i}\right)\right)^{k} \phi(x, y) \text { pour } k \in \mathbf{N} .
$$

Donc, toute fonction $\Delta_{0}$ - définissable et totale dans I $\Delta_{0}$ est bornée par un polynôme. L'intérêt de I $\Delta_{0}$ tient en ceci : certaines preuves en théorie des nombres utilisent des fonctions de croissance non-polynomiale et ne peuvent pas être traduites en preuves à l'intérieur de $I \Delta_{0}$. Cependant, l'énoncé de certains résultats - il s'agit ici d'énoncés $-\Pi_{1}^{0}$ - ne fait pas mention de ces fonctions et on cherche à savoir si ces énoncés sont prouvables dans $I_{\mathbf{0}}$.

Bennett ${ }^{33}$ a donné une définition- $\Delta_{0}$ du graphe de $x^{y}=z$, possédant toutes les propriétés algébriques de l'exponentiation à une exception près puisqu'on ne peut pas, d'après le résultat de Parikh, prouver dans $I \Delta_{0}$ que cette fonction est totale. En ajoutant à $I \Delta_{0}$ l'axiome exp qui dit que

$$
\forall x \forall y \exists z\left(x^{y}=z\right)
$$

on obtient le système $\mathrm{I} \Delta_{0}+\exp$ qui permet d'effectuer la plupart des preuves en théorie élémentaire des nombres (celles du livre de Hardy et Wright, An Introduction to the Theory of Numbers ${ }^{34}$, par exemple). Ce système est équivalent, entre autre, au système de l'arithmétique des fonctions élémentaires

32. R. Parikh, «Existence and Feasibility in Arithmetic », Journal of Symbolic Logic 36, 1971, p. 494-508.

33. J. Bennett, « On Spectra ", thèse de doctorat, Université de Princeton, 1962.

34. G. H. Hardy \& E. M. Wright, An Introduction to the Theory of Numbers, deuxième édition, Oxford, Clarendon Press, 1938. 
(c'est-à-dire à $\mathrm{IE}_{0}$ auquel on ajoute les équations qui définissent les fonction élémentaires de Kalmar). Il s'agit donc de chercher à éliminer l'axiome exp des preuves des énoncés $-\Pi_{1}^{0}$ de $L$ dans $I \Delta_{0}+\exp$. L'intérêt de $I \Delta_{0}$ et de ${ }_{1} \Delta_{0}+\Omega_{1}$ tient aussi aux relations avec la théorie de la complexité computationelle. En particulier, une réponse positive à la question « est-ce que le théorème de Matijasevic-Robinson-Davis-Putnam est démontrable dans $I \Delta_{0}$ (ou dans $\left.\mathrm{I} \Delta_{0}+\Omega_{1}\right)$ ? " entrânerait une solution positive d'un de ses principaux problèmes : $N P=\operatorname{co}-N P$ (avec $N P$ la classe des calculs en temps polynomial « indéterministe », c'est-à-dire que dans certains états de la machine il y a plus d'une action possible, et co-NP la classe des compléments de $N P)^{35}$.

On ne peut cependant pas faire grand chose dans $I \Delta_{0}$, qui n'a que les ressources pour coder les computations en temps linéaire et manque donc de flexibilité : à cause de l'accroissement de la longueur que la substitution introduit, il faut un système qui soit clos pour la multiplication afin de pouvoir parler d'elle. C'est pour cela que l'on s'intéresse à $I \Delta_{0}+\Omega_{1}$, qui permet d'arithmétiser adéquatement la syntaxe ${ }^{36}$. Cet argument est plutôt technique, mais Nelson croit avoir une justification finitiste, sur laquelle je reviendrai dans un instant.

Pour les relations $\omega_{n}(x)=y$, l'énoncé

$$
\forall x \exists y \quad \omega_{n}(x)=y
$$

de $L$, qui exprime le fait que $\omega_{n}$ est totale est dénoté par $\Omega_{n}{ }^{3}$. L'expression $\Omega_{1}$ ou

$$
\forall x \exists y \quad \omega_{1}(x)=y,
$$

(bien sûr, il ne faut pas confondre ce $\omega_{1}$ et l'ordinal $\omega$ de la théorie des ensembles), tient lieu de l'énoncé :

$$
\forall x \exists y\left(y=x^{\log x}\right),
$$

où la fonction $\left(y=x^{\log x}\right)$ possède une définition $-\Delta_{0}$. Notons en passant qu'on ne peut pas prouver que l'exponentiation est totale dans $I \Delta_{0}+\Omega_{1}$, tout comme

35. Cf. H. Gaifman \& C. Dimitracopoulos, «Fragments of Peano's Arithmetic and the MRDP Theorem », dans Logic and Algorithmic, Monographie de L'enseignement mathématique, Genève, 1982, p. 187-206.

36. Voir les explications de A. J. Wilkie \& J. B. Paris dans « On the Scheme of Induction for Bounded Arithmetical Formulas » (Annals of Pure and Applied Logic 35, 1987, p. 264-265) ou de P. Hàjek \& P. Pudlàk dans Metamathematics of FirstOrder Arithmetic (op. cit., p. 273).

37. Pour plus de détails sur ces relations, voir A. J. Wilkie \& J. B. Paris « On the Scheme of Induction for Bounded Arithmetical Formulas », op. cit., p. 264. 
on ne peut pas prouver que la fonction $\left(y=x^{\log x}\right)$ est totale dans $I_{0}$. On a donc en particulier les inclusions strictes suivantes, au début de la hiérarchie des systèmes de $\mathrm{I} \Delta_{0}$ :

$$
\mathrm{I} \Delta_{0} \subset \mathrm{I} \Delta_{0}+\Omega_{1} \subset \mathrm{I} \Delta_{0}+\exp
$$

Il ne faut pas s'inquièter du fait que $\Omega_{1}$ soit un énoncé $\forall \exists$. Il s'agit d'un énoncé- $\Pi_{1}^{0}$ qui porte sur l'ensemble $\omega$ des nombres naturels. On n'ouvre pas la porte aux nombres transfinis, de $\omega$ à $\varepsilon_{0}$. Tout en admettant qu' on peut monter très haut dans la hiérarchie des systèmes $\mathrm{I} \Delta_{0}$ - beaucoup plus haut que $\mathrm{I} \Delta_{0}+\exp$, comme on va le voir - je maintiens qu'il n'y a rien au niveau $\Delta_{0}+\Omega_{1}$ qui nous engage à admettre l'existence d'un ordinal transfini $\alpha<\varepsilon_{0}$. Pour essayer de fixer les idées, revenons au résultat de Parikh mentionné ci-haut. Dans l'antécédent, $\phi(x, y)$ est $\Delta_{0}$, ce que j'indique en soulignant :

$$
\mathrm{I} \Delta_{0}+\forall x \exists y \phi(x, y) \text {. }
$$

Il faut voir $\phi(x, y)$ comme étant l'équivalent des « énoncés réels » de Hilbert, dans « Über das Unendliche ${ }^{38}$ ". En bomant le quantificateur existentiel, le résultat de Parikh étend, façon de parler, la partie « réelle » (soulignée) :

$$
\mathrm{I} \Delta_{0}+\forall x \exists \underline{\exists}, \phi(x, y),
$$

puisque la fonction témoin (witness function) du quantificateur existentiel est computable en temps linéaire. Les ordinaux transfinis n'entrent pas en jeu ici.

L'usage du quantificateur universel ne m'apparaît pas problématique dans ce contexte, puisqu' on peut tout aussi bien considérer la variable $x$ comme libre. Il faut en effet regarder l'expression $\forall \exists$ comme faisant partie de l'usage du symbole de fonction, puisque $\Omega_{1}$ peut être remplacé par le signe de fonction \# (sharp) :

$$
\#(x, y)=x^{\log y}
$$

38. D. Hilbert, «Über das Unendliche », Jahresbericht der Deutschen MathematikerVereinigung 36, 1927, p. 201-215; traduction anglaise, “ On the Infinite », dans J. van Heijenoort (dir.) From Frege to Gödel. A Sourcebook in Mathematical Logic, 1879-1931, Cambridge Mass., Harvard University Press, 1967, p. 367-392. On doit au jeune André Weil une traduction du texte de Hilbert, "Sur l'infini » (Acta Mathematica 48, 1926, p.91-122), qui est malheureusement tombée aux oubliettes. Gauthier sera heureux d'y découvrir que Weil traduisait déjà l'expression hilbertienne " inhaltliche logische Schliessen" par " logique interne de la déduction (p. 101), ce qui est beaucoup mieux que le néologisme «contentuelle » ou que l'expression malheureuse : « raisonnement logique à contenu », utilisés par Jean Largeault dans sa traduction (« Sur l'infini », dans J. Largeault (dir.), Logique mathématique. Textes, Paris, Armand Colin, 1972, p. 220-245). 
Il suffit donc d'enrichir le langage de $L$ à $L^{\#}=\{0, S,+, \cdot \#, \leq\}$. On a donc le système $I \Delta_{0} \#$, qui est axiomatisé par le schème d'induction avec quantificateurs bornés par des termes dans \# et, en plus des axiomes usuels, par des axiomes spécifiques pour \#. On peut aisément prouver que $I \Lambda_{0}^{\#}$ est interprétable dans $I \Delta_{0}+\Omega_{1}$ et vice-versa. Donc, si il y a lieu de s'inquièter à propos de $\Omega_{1}$, c'est plutôt toute la hiérarchie des systèmes de $I \Delta_{0}$ qu'il faut remettre en question : en effet, le système I $\Delta_{0}$ lui-même est axiomatisé par des énoncés $-\Pi_{1}^{0}$ ! On a qu'à regarder le schème d'induction $I \Delta_{0}$ pour voir qu'il possède des quantificateurs universels non-bornés : il s'agit uniquement de restreindre - et c'est là toute une restriction - l'induction à la classe des formules qui ne possèdent que des quantificateurs bornés. Mais cette induction, Gauthier veut la chasser pour la remplacer par la descente infinie.

La position de Gauthier m'apparaît paradoxale : d'un côté il se doit de considérer I $\Delta_{0}$ insuffisant, puisqu'on y prouve moins que dans l'arithmétique de Fermat. D'un autre côté, Gauthier considère $I \Delta_{0}$, avec son induction, comme un cadre formel infinitaire, donc beaucoup moins « restreint». À quoi doit-on s'en tenir ? Peut-être doit-on simplement s'arrêter ici : l'arithmétique de Fermat, avec son quantificateur effini et sa descente infinie peut-elle vraiment être comparée avec la hiérarchie des sous-systèmes de l'arithmétique de Peano ? S'il est question de discréditer l'arithmétique de Peano, alors toute restriction de l'induction à des classes de formules telles que $\Delta_{0}$ ne serait que peine perdue. Cependant, Nelson a tenté de donner une justification génétique

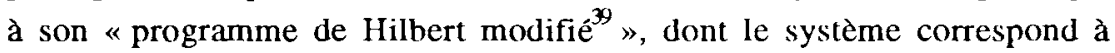
$\mathrm{Q}+\left(\mathrm{I} \Delta_{0}+\Omega_{1}\right)$; ce qui le distingue de l'arithmétique de Peano. C'est celui-ci et non pas l'ensemble de la hiérarchie $I \Delta_{0}$, que j'aimerais comparer à l'arithmétique de Fermat.

De l'avis de Nelson, que je partage, l'induction n'est pas dangereuse dans le contexte de $I \Delta_{0}+\Omega_{1}$ : si problème il y a, il est situé au-delà, avec les fonctions à croissance plus rapide telles que l'exponentiation. En effet, même si $\Omega_{1}$ dit que la fonction $y=x^{\log x}$ est totale, cette fonction reste computable en temps polynomial. Notons que la fonction sharp est étroitement reliée à la fonction \# (smash) de Sam Buss

$$
x \# y=2^{|x|} \cdot|y|,
$$

qui possède la rapidité de croissance nécessaire pour définir les fonctions

39. E. Nelson, Predicative Arithmetic, op. cit., chapitres 1, 18 et 30-32. 
computables en temps polynomial ${ }^{40}$. L'expression $\Omega_{1}$ peut donc être remplacée par l'adjonction de la fonction \# qui est computable en un temps polynomial, tout comme $+\mathrm{et} \cdot$, tandis que ce n'est pas le cas avec l'exponentiation. Il y a donc une barrière à franchir avec l'exponentiation. C'est cette «barrière infranchissable », comme le dit Nelson, qui cause problème, si problème il y a. Voilà donc une bonne raison pour associer $I \Delta_{0}+\Omega_{1}$ avec $I \Delta_{0}$ plutôt qu'avec $\mathrm{I} \Delta_{0}+\exp$.

Venons-en finalement au " programme de Hilbert modifié " proposé par Nelson. Le point de départ de Nelson dans Predicative Arithmetic est le système $\mathbf{Q}$ de Raphael Robinson ${ }^{41}$, qui n'est que l'arithmétique de Peano sans l'induction. On n'a même pas le

$$
\Xi x(S x=x+1)
$$

de Gauthier (p. 99), mais simplement

$$
(x \neq 0) \rightarrow \exists y(\mathrm{~S} y=x)
$$

où le quantificateur existentiel peut-être éliminé en introduisant la fonction de différence modifiée (introduite à l'origine par Skolem ; il s'agit de la fonction $\delta$ de Kronecker) :

$$
(x \neq 0) \rightarrow \mathrm{S}(x \dot{-})=x .
$$

Nelson et Wilkie ont démontré qu'il y a une définition uniforme dans les modèles de $Q$ d'un segment initial qui est un modèle de $I \Delta_{0}+\Omega_{1}$. Pour Nelson, il s'agit là d'une justification finitiste, puisque le système $\mathbf{Q}$ de Robinson est tout simplement irréprochable à cet égard ; à moins que l'on se place du point de vue finitiste strict selon lequel même l'addition et la multiplication ne sont pas des fonctions totales, puisqu'une borne supérieure à l'activité du mathématicien est postulée. Je me rappelle d'une paraphrase de Kronecker par Robin Gandy, en conversation, qui illustre bien la différence de points de vue : «selon Nelson, Dieu créa l'addition et la multiplication, le reste n'est que l'oeuvre des hommes ». Je reviendrai sur le finitisme strict dans la section 3.

Pour continuer sur un ton informel, l'idée maîtresse de Nelson consiste à

40. S. R. Buss, Bounded Arithmetic, op. cit.

41. R. Robinson, «An Essentially Undecidable Axiom System », dans Proceedings of the International Congress of Mathematicians, 1950, Providence R. I., American Mathematical Association, 1952, p. 729-730, et E. Nelson, Predicative Arithmetic, op. cit., chapitre 3 . 
prendre un modèle $\mathbf{M}$ de $\mathrm{I} \Delta_{0}$, et à en considérer le segment initial $\mathbf{B}$, appelé « segment de Bennett" :

$$
x \in \mathbf{B} \leftrightarrow(\exists y)\left(y=2^{x}\right),
$$

qui est clos pour + et $\cdot$ si on a $\Omega_{1}$. Comme l'a remarqué Macintyre, ce domaine "a beaucoup en commun avec $\omega^{42}$ ". L'analogie a ceci d'intéressant : on peut effectuer une analyse non standard (de type « interne » et non pas à la Robinson) à justification finitiste, sur laquelle je reviendrai dans la section 4. À supposer que ce programme s'avère sans faille, on pourrait alors développer une grande partie de l'analyse à l'intérieur des extensions de $\mathbf{Q}$, un résultat qui serait pour le moins spectaculaire. Ce programme est malheureusement un peu mal pensé (par exemple, le quantificateur $\exists$ ci-dessus est nonborné) : Nelson n'a pas fait attention au fait que la démonstration de l'interprétabilité de $I \Delta_{0}+\Omega_{1}$ dans $Q$ fait usage de quantificateurs non-bomés, comme le lui a reproché, entre autre, Pavel Pudlàk ${ }^{43}$. La question : «est-ce qu'une démonstration d'interprétabilité de $I \Delta_{0}+\Omega_{1}$ dans $Q$ est possible qui n'utiliserait que des quantificateurs bornés? » reste encore sans réponse. Une réponse négative serait fatale.

Faisant abstraction de ces problèmes, mon objection contre l'arithmétique de Fermat consiste simplement à réclamer pour le point de vue $\mathrm{I} \Delta_{0}+\Omega_{1}$ revu par Nelson - et seulement pour celui-ci, non pas pour le plein point de vue de la hiérarchie des systèmes $I \Delta_{0}$ - un statut finitiste plus fort que celle-ci et à affirmer, peut-être à tort, qu'on ne peut critiquer le point de vue $I \Delta_{0}+\Omega_{1}$ qu'en adoptant une position finitiste stricte que Gauthier récuse. (Sur ce dernier point, voir le début de la section suivante.) Il est important de rappeler que Nelson a un théorème « d'auto-consistance génétique ${ }^{44}$ » et qu'il n'a donc pas besoin de l'induction transfinie pour la justification de son système.

Afin de prévenir une objection potentielle, je dois mentionner les travaux de Richard Sommer, qui a étendu la notion d'ordinal $\alpha<\varepsilon_{0}$ aux modèles de I $\Delta_{0}$ afin d'y démontrer inter alia certains résultats élémentaires sur les ordinaux ${ }^{45}$. Sommer a en effet montré que l'on peut « faire » une partie importante

42. A. Macintyre, « The Strength of Weak Systems », op. cit., p. 51.

43. P. Pudlàk, "Review of E. Nelson : Predicative Arithmetic ", Joumal of Symbolic Logic 53, 1988, p. 987-989.

44. E. Nelson, Predicative Arithmetic, op. cit., p. 172, théorème 30.27.

45. R. Sommer, * Transfinite Induction and Hierarchies Generated by Transfinite Recursion within Peano Arithmetic », thèse de doctorat, Université de la Californie à Berkeley, 1990. 
de l'arithmétique ordinale dans $\mathrm{I} \Delta_{0}$ et I $\Delta_{0}+\Omega_{1}$. Mais il ne faut pas se méprendre sur la signification de ces résultats. Si le modèle de $\mathrm{I} \Delta_{0}$ et $\mathrm{I} \Delta_{0}+\Omega_{1}$ reste l'ensemble $\omega$ des nombres naturels, la métathéorie de Sommer, ZFC (la théorie des ensembles de Zermelo-Fraenkel avec l'axiome du choix), a pour modèle des ensembles infinis (les ordinaux $\alpha<\varepsilon_{0}$ étant des ensembles infinis). Supposons maintenant que $\mathbf{R}$ est l'ensemble des symboles de relation ordinales usuelles, et $\mathbf{F}$ l'ensemble des symboles de fonction ordinales usuelles. Sommer démontre que pour $L^{*}=\{(0, S,+, \cdot, \leq) \cup \mathbf{R} \cup \mathbf{F}\}$, on a un codage (Gódel numbering)

$$
\alpha \Rightarrow[\alpha]
$$

pour les ordinaux $\alpha<\varepsilon_{0}$, et un ensemble $D$ d'énoncés-L* tels que

[ ] est une bijection de $\varepsilon_{0}$ dans un sous-ensemble de $\omega$.

De plus, pour chaque relation $\mathrm{R}$ de $\mathbf{R}$, on a :

$$
\forall x\left(\mathrm{R}(x) \leftrightarrow \theta_{\mathrm{R}}(x)\right),
$$

avec $\theta_{R} \in \Delta_{0}$; et pour chaque fonction $\mathrm{F}$ de $\mathbf{F}$, on a :

$$
\forall \boldsymbol{x}, y\left(\mathrm{~F}(\boldsymbol{x})=y \leftrightarrow \theta_{\mathrm{F}}(\boldsymbol{x}, y)\right),
$$

avec $\theta_{\mathrm{F}} \in \Delta_{0}$, et, au besoin,

$$
\mathrm{I} \Delta_{0}+\Omega_{1}+\forall x \exists ! y \theta_{\mathbf{F}}(x, y) ;
$$

etc... Ce résultat permet de dire que, pour la plupart des fonctions, les " ordinaux » dans le modele de $\mathrm{I} \Delta_{0}$ correspondent aux ordinaux dans ZFC.

Le fait qu'on puisse coder les ordinaux transfinis dans $I \Delta_{0}$ ne semble pas avoir d'intérêt philosophique : il est vrai, d'un côté, que I $\Delta_{0}$ possède les ressources pour un tel codage (une variante du codage de Smullyan) mais il faut, de l'autre côté, accepter le langage $\mathrm{L}^{*}$ et se placer dans la métathéorie ZFC. (Il faut aussi se demander si de toute façon l'arithmétique de Fermat ne possède pas elle-même les ressources pour coder les ordinaux transfinis. Une réponse négative n'est pas évidente.) Quant à l'induction transfinie, elle n'apparait pas dans $I \Delta_{0}$. Il faut l'ajouter : Sommer travaille avec $I \Delta_{0}+\{T I(\alpha$, $\varphi): \varphi \in C$ \} , où $T K(\alpha, \varphi)$ dénote l'induction transfinie jusqu'à $\alpha$. Ces travaux ne font donc sens que si on monte dans la hiérarchie des systèmes de $I_{0}$ au moins jusqu'au niveau de la fonction d'Ackermann $F_{\omega}$, qui est définie par diagonalisation sur les fonctions : 


$$
\begin{gathered}
\mathrm{F}_{0}(x)=x+1 \\
\mathrm{~F}_{n+1}(x)=\mathrm{F}_{n}^{(x+1)}(x),
\end{gathered}
$$

(pour tout $n \in \mathbf{N}$ ). On peut non seulement obtenir une définition - $\Delta_{0}$ du graphe de $\mathrm{F}_{\omega}$ - et donc concevoir le système $\mathrm{I} \Delta_{0}+\mathrm{F}_{\omega}$ - mais encore une définition $-\Delta_{0}$ des graphes des fonctions de la hierarchie de Wainer, qui présupposent un système de notation ordinale jusqu'à $\varepsilon_{0}$. Mais, encore une fois, avec ces fonctions nous sommes bien loin du domaine des fonctions computables en temps polynomial et du segment de Bennett : on ne respecte plus le point de vue génétique adopté par Nelson.

\section{Entre le finitisme strict et l'arithmétique sans quantificateurs.}

Il ne faut pas oublier qu'il y a un point de vue fondationel encore plus faible que celui de $I \Delta_{0}+\Omega_{1}$, soit le finitisme strict associé aux travaux de Esenin-Volpin $^{46}$ et, plus récemment, à des auteurs tels que Robin Gandy, Vladimir Sazonov et bien d'autres ${ }^{47}$. En effet, le point de vue $I \Delta_{0}+\Omega_{1}$ se distingue par le rôle central joué par la notion de computabilité en temps polynomial. Pour Buss, par exemple, "faisabilité " est synomyne de " computabilité en temps polynomial ». En théorie de la complexité computationelle, l'intérêt se porte sur les bornes supérieures à la quantité de ressources computationelles utilisées par un algorithme qui résout un problème décidable. Il y a deux mesures de ces ressources, le temps, c'est-à-dire le nombres d'étapes exécutées par l'algorithme, et l'espace, c'est-à-dire la quantité de

46. A. S. Esenin-Volpin, "Le programme ultra-intuitionniste des fondements des mathématiques ", dans Infinitistic Methods. Proceedings of the Symposium on the Foundations of Mathematics, September 1959, Warsaw, Varsovie, PWN Warszawa, 1961, p. 201-223, "The Ultra-Intuitionistic Criticism and the AntiTraditional Program for the Foundations of Mathematics », dans J. Myhill, A. Kino \& R. E.Vesley (dir.), Intuitionism and Proof Theory, Amsterdam, NorthHolland, 1970, p. 3-45 ; et "About Infinity, Finiteness and Finitization ", dans F. Richman (dir.), Constructive Mathematics. Proceedings of the New Mexico State University Conference 1980, Berlin, Springer, Lecture Notes in Mathematics vol. 873,1981 , p. 274-313.

47. Le texte de Robin Gandy, «Limitations to Mathematical Knowledge " (D. van Dalen, D. Lascar \& T. J. Smiley (dir.), Logic Colloquium '80, Amsterdam, NorthHolland, 1982, p. 129-146) constitue une excellente introduction au finitisme strict. 
mémoire utilisée par l'algorithme. Une borne supérieure sur le temps requis est donnée en exhibant un algorithme et en bornant ses ressources, et si un problème donné peut être résolu en un temps $c n^{k}$ pour des constantes $c$ et $k$, où $n$ est la longueur du input, alors l'algorithme est computable en temps polynomial. Aucune restriction n'est cependant émise à propos de la longueur de $n$.

Je prends ici comme exemple l'élimination des quantificateurs pour le fragment $\{0, S,+, \leq\}$, connu sous le nom d'arithmétique de Prezburger ${ }^{48}$. Les techniques en théorie des modèles telles que la model-completeness d'Abraham Robinson ou celle des ultraproduits ne permettent d'obtenir que des algorithmes récursifs généraux, tandis qu'avec l'élimination des quantificateurs (qui est d'origine kroneckerienne) on obtient des algorithmes récursifs primitifs. On peut obtenir pour ceux-ci des bornes temporelles pour leur complexité, mais celles-ci sont trop larges pour être « faisable »: les meilleures bornes dans la littérature sont des itérations finies de la fonction exponentielle. Par exemple, la meilleure borne supérieure connue pour l'arithmétique de Prezburger est $^{49}$ :

$$
2^{2^{c n}}
$$

Comme je l'ai dit, il n'y a pas de restriction sur la longueur de $n$. On peut cependant ajouter une restriction supplémentaire et postuler qu'il y a une borne supérieure à la longueur de toute communication entre mathématiciens. En tenant compte de l'existence de celle-ci, on peut construire des modèles totalement finis de l'arithmétique, comme tente de le faire Robin Gandy, par exemple $^{50}$. Pour ce point de vue finitiste strict les nombres finis très grands sont équivalent à l'infini, ce que Vladimir Sazonov ${ }^{\text {sl }}$ exprime en disant que de son point de vue :

$$
2^{2^{10}}=2^{1024}=\infty
$$

48. M. Prezburger, "On the Completeness of a certain System of Arithmetic of Whole Numbers in which Addition occurs as the only Operation ", History and Philosophy of Logic 12, 1991, p. 225-233.

49. J. Ferrante \& C. W. Rackoff, The Computational Complexity of Logical Theories, Berlin, Springer, Lecture Notes in Mathematics 718, 1979, p. 47-54

50. R. O. Gandy, "Completely Finite Models for Number Theory », Journal of Symbolic Logic 54, 1989, p. 653, et « Totally Finite Interpretation of First-Order Arithmetic. (Second Draft)», manuscrit, 1989.

51. V. Yu. Sazonov, « On Feasible Numbers », Joumal of Symbolic Logic 57, 1992, p. 331 . 
Gauthier écarte lui-même ce point de vue finitiste strict (p. 75, note 4) auquel il associe Esenin-Volpin et Wittgenstein. (Je reviendrai sur l'interprétation de Wittgenstein dans la section 5.) Je ne suis pas convaincu, cependant, que le constructivisme radical de Gauthier ne le force pas à adopter des positions finitistes strictes. En effet, j'ai tenté d'indiquer dans la section précédente que si on prend au sérieux sa volonté de vouloir mettre sur pied avec son arithmétique de Fermat, un système plus « restreint » que ceux associés à $I \Delta_{0}+\Omega_{1}$, il est forcé à mon avis d'adopter une position finitiste stricte. Sinon, il doit reconnaître que son arithmétique n'est pas aussi faible qu'il voudrait bien qu'elle soit.

Un autre question à propos de laquelle Gauthier s'engage pratiquement à adopter une thèse finitiste stricte est celle de l'imprédicativité. Gauthier qualifie l'imprédicativité de la définition logiciste des nombres naturels de « rédhibitoire » (p. 82). C'est un fait bien connu que les définitions de Frege et Dedekind au second-ordre sont imprédicatives. On peut cependant se demander si une certaine dose d'imprédicativité n'est pas nécessaire dans tout raisonnement mathématique. En effet, Dummett a fait, il y a déjà longtemps, la remarque selon laquelle une forme d'imprédicativité reste présente même si, afin d'éviter le second-ordre plein, on traite l'induction non pas comme une définition, mais comme une partie de l'explication informelle du prédicat «nombre naturel ${ }^{52}$ ". Il semble que la notion de nombre naturel soit déjà imprédicative. On retrouve ici le fameux cercle vicieux dénoncé par les finitistes stricts. Les numéraux sont habituellement définis comme suit : 0 est un numéral et si $n$ est un numéral, alors $S n$ est un numéral. Les numéraux sont donc représentés par la suite

$$
0, \text { SO, SSO, SSSO, SSSSO, ... }
$$

Selon ce raisonnement, on peut, par cette suite atteindre n'importe quel nombre naturel. La vérité du schéma d'induction $\mathrm{I}(\phi)$ de Peano repose précisément sur notre reconnaissance de ce fait. Esenin-Volpin avait à l'époque pointé du doigt la circularité de cette explication, en considérant l'assertion

$$
[\phi(0) \wedge \forall x(\phi(x) \rightarrow \phi(x+1))] \rightarrow\left(\phi\left(10^{12}\right)\right) .
$$

Pour Esenin-Volpin, il est physiquement impossible d'utiliser $10^{12}$ fois le Modus Ponens, puisqu'en secondes $10^{12}$ équivaut à plus de 20000 ans. Mais d'après les explications précédentes on atteint le $10^{12}$-ième membre de la

52. M. A. E. Dummett, «The Philosophical Significance of Gödel's Theorem », dans M. A. E. Dummett, Truth and other Enigmas, Londres, Duckworth, 1978, p. 199. 
suite des nombres naturels en $10^{12}$ étapes. Or l'expression « $n$ étape » présuppose que $n$ soit un nombre naturel. On voit bien la circularité de la conception traditionelle des nombres naturels. Cette circularité n'est pas moins vicieuse, selon Esenin-Volpin, « que celle que l'on retrouve dans les définitions imprédicatives de la théorie des ensembles ${ }^{53}$ ". On fait donc face ici à un dilemme : soit on nie que l'imprédicativité soit vicieuse et on ne peut plus alors parler de vice rédhibitoire (Charles Parsons ${ }^{54}$, par exemple, adopte ce point de vue à la suite de Dummett), soit on va de l'avant, comme le font les finitistes stricts depuis Esenin-Volpin, et on remet en question la notion même de « nombre naturel », c'est-à-dire qu'il faut rejeter l'unicité de la suite des nombres naturels et il $n^{\prime} y$ a plus d'isomorphie ${ }^{55}$. Si Gauthier veut que la critique prédicativiste conserve son mordant, il me semble contraint d'opter pour la seconde alternative. Cela convient mieux à son constructivisme, qui est plus radical que celui d'un Dummett ; ce dernier étant prêt à accepter une certaine dose d'imprédicativité tandis que pour Gauthier comme pour Nelson, « le raisonnement finitaire imprédicatif est un résidu de platonisme qui a été accepté par les finitistes sans faire preuve d'esprit critique ${ }^{56}{ }^{\text {} . ~ C ' e s t ~ u n e ~ a n a l y s e ~ d e ́-~}$ taillée de la notion de prédicativité qu'il nous faut pour compléter le point de vue de Gauthier, qui me semble bien contraint à adopter une position finitiste stricte.

Gauthier se doit aussi de distinguer son arithmétique de Fermat de l'arithmétique de Skolem, et du même coup du calcul équationel de Louis

53. A. S. Esenin-Volpin, "The Ultra-Intuitionistic Criticism and the Anti-Traditional Program for the Foundations of Mathematics», op. cit., p. 5. On trouve un argument tout à fait similaire dans R. Parikh, « Existence and Feasibility in Arithmetic », op. cit., p. 502-503, ainsi que dans E. Nelson, Predicative Arithmetic, op. cit., p. 174, et, encore plus récemment, dans J. Mycielski, « The Meaning of Pure Mathematics ", Joumal of Philosophical Logic 18, 1989, p. 316. Le chapitre 18 de Predicative Arithmetic contient un important résultat à l'appui de cette critique.

54. C. Parsons, «The Impredicativity of Induction », dans M. Detlefsen (dir.), Proof, Logic and Formalization, Londres, Routledge, 1992, p. 139-161.

55. Sur ce sujet, je conseille la lecture de D. Isles, « Remarks on the Notion of Standard Non-Isomorphic Natural Number Series», dans F. Richman (dir.), Constructive Mathematics. Proceedings of the New Mexico State University Conference 1980, op. cit., p. 111-134.

56. E. Nelson, Predicative Arithmetic, op. cit., p. 177. On notera que ce point de vue rend erronée la thèse de Bill Tait selon laquelle « il ne peut pas y avoir de point de vue préférable à partir duquel on puisse lancer une attaque contre le finitisme " ( Finitism », Journal of Philosophy 78,1981, p. 546). 
Goodstein, qui sont un peu les ancêtres de l'arithmétique bornée ${ }^{57}$. Ici, nous sommes réduit à l'affirmation de Gauthier selon laquelle l'arithmétique de Fermat " est la seule arithmétique qui ne soit pas ensembliste, i.e. qui ne fasse pas appel à un ordinal-limite infini - même l'arithmétique récursive de Skolem [...] ne saurait s'en passer » (p. 116). Ce propos m'apparaît ne pas être tout à fait juste. Tout d'abord, Gauthier passe sous silence la general set theo$r y$, c'est-à-dire la théorie des ensembles de Zermelo-Fraenkel sans l'axiome d'infinité. Cette théorie, qui reste malheureusement peu étudiée ${ }^{58}$, peut être interprétée sur les ensembles héréditairement finis (c'est-à-dire les ensembles qui sont finis, dont tous les éléments sont finis, tous les éléments des éléments sont finis, etc...), et elle est bien plus forte qu'elle ne paraît à prime abord, puisqu'on peut y interpréter l'arithmétique de Peano. John Mayberry a récemment défendu cette théorie générale des ensembles ${ }^{59}$, qui correspond selon lui à l'arithmétique des fonctions élémentaires de Kalmar, d'un point de vue finitiste qui se rapproche de celui de Esenin-Volpin (sans pour autant faire usage des étranges notions temporelles et modales de celui-ci). L'abandon de unicité de la suite des nombres naturels est une des conséquences de l'approche de Mayberry ${ }^{60}$. D'un autre côté, l'abandon de l'axiome d'infinité n'implique pas l'abandon total de l' approche ensembliste.

Bill Tait ayant émis la thèse couramment acceptée selon laquelle les fonctions finitistes sont exactement les fonctions récursives primitives ${ }^{61}$, l'arithmétique de Skolem joue un rôle important dans les discussions sur le finitisme. Le but avoué de Skolem dans son fameux texte de 1923 était de ne pas utiliser les quantificateurs non-bornés ${ }^{62}$. Le «mode de pensée récursif »

57. Le système PV de Cook et Urquhart ( « Functional Interpretations of Feasibly Constructive Arithmetic », op. cit.) est un bon exemple. Dans « Feasibly Constructive Proofs and the Propositional Calculus " (Conference Record of the Seventh Annual ACM Symposium on Theory of Computing, New York, Association for Computing Machinery (ACM), 1975, p. 83-97), Cook a introduit des fonctions computables en temps polynomial par des équations de récursion dans le style de Skolem et Goodstein, auxquels il fait explicitement référence. À la différence du système de Skolem, PV n'a de symboles que pour les fonction computables en temps polynomial.

58. Le locus classicus reste H. Wang, A Survey of Mathematical Logic, Pékin/ Amsterdam, Science Press/North Holland, 1963, p. 480-489.

59. J. Mayberry, "What are Numbers?", Philosophical Studies 54, 1988, p. 317 354.

60. Ibid., p. 350.

61. W. Tait, « Finitism », op. cit., p. 533.

62. T. Skolem, « The Foundations of Elementary Arithmetic established by means of 
proposé par Skolem correspond à l'usage de définitions récursives primitives pour l'introduction de nouvelles fonctions, de quantificateurs bornés uniquement comme abréviation dans les cas finis et de variables libres lorsque le domaine est non-fini. Si Skolem utilise l'induction mathématique, le calcul équationel de Louis Goodstein s'en passe totalement, tout comme il se passe du calcul propositionel au grand complet ${ }^{63}$. Toutes les propositions sont des équations de la forme $\mathrm{A}=\mathrm{B}$ où $\mathrm{A}$ et $\mathrm{B}$ sont des fonctions ou des termes primitifs récursifs et où les règles sont la substitution et l'unicité - cette demière remplaçant l'induction, selon une idée de Dedekind et Wittgenstein. Comme Goodstein le dit lui-même, le seul infini est la possibilité « illimitée » ou indéfinie de remplacer une variable numérique par un numéral (numeral) précis ou par un symbole de fonction ${ }^{64}$. Comme le dit Goodstein, le calcul équationel «ne fait pas appel au schéma d'induction ${ }^{65}$ ». Il n'était pas question pour Skolem (ni pour Goodstein) d'introduire $\omega$, malgré qu'il soit vrai qu'en théorie de la preuve on associe l'ordinal $\omega^{\omega}$ comme borne à l'arithmétique de Skolem. Gauthier pourrait y voir l'indication d'un manque de constructivité. On peut cependant renverser ce raisonnement et y voir que ce qui apparaît at face value comme non-constructif ne l'est pas vraiment et ne présuppose pas des totalités infinies. Gauthier doit s'adresser à ce genre d'argument, sur lequel est fondée une bonne partie de la théorie de la preuve contemporaine, que ce soit les travaux de Kreisel, de Feferman, de l'école allemande de Schütte, ou encore la Reverse Mathematics de Friedman.

Le point de vue de Gauthier s'oppose diamétralement à celui de Kreisel, qui critique la thèse de Tait pour des raisons opposées ${ }^{66}$. Pour Gauthier, c'est

the Recursive Mode of Thought, without Use of Apparent Variables ranging over Infinite Domains ", dans J. van Heijenoort (dir.), From Frege to Gödel, op. cit., p. 304.

63. R. L. Goodstein, Constructive Formalism, Leicester, Leicester University Press, 1951, et Recursive Analysis, Amsterdam, North-Holland, 1957.

64. R. L. Goodstein, Constructive Fomalism, op. cit., p. 52.

65. R. L. Goodstein, Development of Mathematical Logic, Londres, Logos Press, 1971, p. 113. Pour Gauthier cette possibilité illimitée correspond cependant à l'induction complète ( « Finite Arithmetic with Infinite Descent», Dialectica 43, 1989, p. 336).

66. G. Kreisel, «Ordinal Logics and the Characterization of Informal Concepts of Proofs ", dans, J. A. Todd (dir.), Proceedings of the International Congress of Mathematicians, Cambridge, Cambridge University Press, 1958, p. 289-299, et « Principles of Proof and Ordinals implicit in given Concepts», dans J. Myhill, A. Kino \& R. E.Vesley (dir.), Intuitionism and Proof Theory, op. cit., p. 489-516. 
non seulement la notion de récursion transfinie, mais aussi la notion de récursion primitive qu'il faut remettre en question. Dans la perspective des logiques ordinales (ordinal logics) ouverte par les travaux de Turing, Kreisel a tenté de circonscrire le domaine des preuves finitistes (à sa suite, Feferman a fait de même pour la prédicativité) et selon lui le domaine du finitisme déborde du domaine des fonctions récursives primitives : les fonctions finitistes sont précisément celles que l'on peut prouver comme existant dans la théorie des nombres du premier ordre. L'argument de Kreisel peut être décrit comme suit : supposons que $\mathrm{F}(x, y)$ est une équation à deux variables et $\mathrm{S} n$ dénote le numéral SSS ... S0 avec $n$ occurences de S. Supposons que l'on a les fonctions finitistes $\phi$ et $\gamma$ telles que l'on peut prouver dans l'arithmétique récursive pimitive que $\gamma(x)$ soit un terme $t$ de celle-ci, et que $\phi(x)$ soit une déduction formelle dans l'arithmétique récursive primitive de $\mathrm{F}(\mathrm{S} x, t)$. Or, puisqu' un finitiste doit reconnaître que $\mathrm{F}(\mathrm{S} n, t)$ est déduit, il doit reconnaitre qu'il y a un nombre $x$ tel que $\mathrm{F}(n, x)$. On peut ainsi introduire la fonction

$$
\eta(n)=\text { « le plus petit nombre } x \text { tel que } \mathrm{F}(n, x) \text { ». }
$$

Kreisel croit donc légitimer de cette façon l'introduction de fonctions nonrécursives telles que $\eta$, d'un point de vue finitiste. Les arguments de Kreisel sont partagés par de nombreux logiciens ${ }^{67}$, et il serait utile que Gauthier précise aussi ses objections envers celui-ci.

\section{Analyse non standard}

Pour revenir au " programme de Hilbert modifié » de Nelson, j'aimerais m'opposer au scepticisme de Gauthier à propos des vertus de l'analyse non standard (ANS), dont l'usage permet une extension remarquable des fragments faibles que nous avons abordés. L'attrait de l'ANS vient de la possibilité de travailler avec deux modèles, en particulier de la possibilité de modèles " hyperfinis ». De prime abord, Gauthier est sceptique, car il semble difficile de donner un modèle non standard du continu sans faire appel à une théorie des ensembles forte. L'ANS d'Abraham Robinson a été renouvelée récemment grâce à l'introduction d'une nouvelle syntaxe par Edward Nelson dans "Internal Set Theory. A New Approach to Nonstandard Analysis ${ }^{68}$ ".

67. Cf. H. E. Rose, Subrecursion. Functions and Hierarchies, Oxford, Oxford University Press, 1984, p. xi.

68. E. Nelson, «Internal Set Theory. A New Approach to Nonstandard Analysis", op. cit. 
S'attaquant à l'approche de Nelson dans le deuxième appendice (p. 112-114), Gauthier qualifie la théorie interne des ensembles (IST) de théorie « hautement imprédicative » (p. 113). On peut bien sûr lui concéder ce point, puisque pour obtenir l'IST il faut tout d'abord se donner la théorie des ensembles de Zermelo-Frankel avec l'axiome du choix (ZFC). Mais l'intérêt de l'ANS n'en est pas diminué pour autant. À mon avis, on peut donner des fondements finitistes à l'ANS en s'aidant des travaux de Nelson lui-même.

L'axiome d'idéalisation joue à mon avis un rôle crucial, puisqu'il en découle que les ensembles infinis standards de IST tel que $\mathbf{N}$ ou $\mathbf{R}$ contiennent des membres non standards ${ }^{69}$. (C'est là une différence essentielle avec l'approche de Robinson : les non standards sont déjà dans l'univers de IST, alors que Robinson doit construire un nouvel ensemble $* \mathbf{R} ; \mathbf{R}$ dans IST correspond à $* \mathbf{R}$ dans une " superstructure ».) On a donc deux modeles, celui des entiers "standards » ou " naîfs » et celui des " non standards" ou " nonnaïs ». Si on se place du point de vue de ZFC, le plus « petit » modèle sera le modèle standard. Il faut tout simplement rejeter ce point de vue et dire que c'est plutôt le modèle non standard qui est le plus " petit ». Pour faire cela, il faut donner une version plus faible ou abandonner les principes de transfert et de standardisation ${ }^{70}$. On peut interpréter ainsi ce que fait Nelson dans le dernier chapitre de Predicative Arithmetic, où il introduit sa théorie $\mathbf{Q}^{*}$. Celle-ci contient son arithmétique prédicative (on dépasse donc le point de vue $I \Delta_{0}+\Omega_{1}$ ) et un prédicat $\phi$ accompagné d'axiomes qui disent que $\phi$ respecte chaque symbole de fonction bomé et chaque axiome non-logique borné. Ce qui a pour conséquence, pour $\mathrm{D}$ bomé, que si $1-\mathrm{Q}^{*} \mathrm{D}$, alors $\mathrm{L}_{\mathrm{Q}^{*}} \mathrm{D}^{\phi}$, ce qui remplace le principe de transfert. Pour ce qui est du principe de standardisation, on pourrait utiliser une version plus faible telle que le principe de

69. Ibid., p. 1167

70. Il faut noter ici, que malgré leur justification intuitionniste, Reeb, Harthong et Diener se permettent le plein point de vue de ZFC. Pour se renseigner sur l'analyse non standard de l'école de Strashourg, le lecteur pourra consulter le manuel, F. Diener \& G. Reeb, Analyse non standard, Paris, Hermann, 1989. Pour la justification intuitionniste, voir G. Reeb, « La mathématique non standard vieille de soixante ans ? ", Strasbourg, Publications de l'Institut de Recherche Mathématique Avancée (IRMA), 1979, et «Intuitionnisme, Formalisme, Mathématique non standard et Infinitésimaux », dans B. Seke \& R. Bebbouchi (dir.), Bakundi (1) 1981-1983. Traces d'un séminaire, Strashourg, IRMA, 1983, p. 13-35, et G. Reeb \& J. Harthong, « Intuitionnisme 1984 », dans H. Barreau \& J. Harthong (dir.), La mathématique non standard, op. cit., p. 213-252. 
récurrence externe de Diener \& Reeb ${ }^{71}$. Le truc c'est que $\phi(x)$ est équivalent à « $x$ est standard » dans IST. La théorie $\mathbf{Q}^{*}$ contient aussi la constante $\mathrm{N}$ avec les axiomes

$$
\varepsilon(2 \uparrow N), \varepsilon(2 \uparrow 2 \uparrow N), \ldots
$$

( $\uparrow$ étant le symbole pour la superexponentiation) et l'axiome $\neg \phi(N)$, qui est équivalent au principe d'idéalisation ${ }^{2}$. Nelson montre aussi que $Q^{*}$ reste interprétable localement dans $\mathbf{Q}$, et il donne un argument, insuffisant cependant, en faveur de sa consistance. Avec cette petite portion de IST - dont la possibilité d'une justification finitiste reste cependant douteuse - Nelson a obtenu une élégante version élémentaire non standard du théorème de de MoivreLaplace dans Radically Elementary Probability Theory ${ }^{73}$. On voit bien, donc, que Nelson n'a pas utilisé le plein point de vue de IST.

C'est sur cette base que réside, à mon avis, l'avenir de la philosophie constructiviste des sciences. En effet, les résultats obtenus depuis quelques années - en particulier ceux de Nelson - sont impressionants, et permettent d'espérer une contructivisation complète de la physique mathématique. Ces remarques sont purement spéculatives et ici aussi, tout reste à faire. Mais on voit bien l'attrait de cette approche ${ }^{74}$ ! Gauthier l'a bien vu et il adopte l'approche syntaxique de Nelson dans un nouvel ouvrage, La logique interne des théories physiques ${ }^{75}$, sans pour autant abandonner sa critique de $\mathbf{Q}^{*}$.

\section{Philosophie de la logique}

L'entreprise métamathématique de la logique interne est de nature révisionniste, tout comme l'est la logique intuitionniste. Lorsqu' on ne veut pas adopter en bloc toutes les mathématiques classiques, il existe bien des façons

71. F. Diener \& G. Reeb, Analyse non standard, op. cit., p. 35.

72. E. Nelson, Predicative Arithmetic, op. cit., p. 179.

73. E. Nelson, Radically Elementary Probability Theory, op. cit., chapitre 18

74. Il faut aussi mentionner les travaux de Jan Mycielski sur ses théories localement finies, qui ouvrent la possibilité d'une ANS purement finitaire. $C f$. J. Mycielski, «Analysis without Actual Infinity ", Journal of Symbolic Logic 46, 1981, p. 625633, et « Locally Finite Theories », Journal of Symbolic Logic 51, 1986, p. 59-62. Une autre avenue est celle de la théorie intuitionniste des types de Martin-Löf. Celui-ci a montré récemment comment développer à partir de celle-ci une ANS intuitionniste dans « Mathematics of Infinity » (P. Martin-Löf \& G. Mints (dir.), COLOG-88, Berlin, Springer, Lecture Notes on Computer Science 417, 1990, p. 146-197).

75. Y. Gauthier, La logique interne des théories physiques, Montréal/Paris, Bellarmin/Vrin, 1992, p. $95 s q$. 
de les découper et ce qu'il faut c'est une motivation philosophique pour justifier le découpage que l'on choisit. Il se peut très bien que celui-ci ne corresponde à aucune position philosophique cohérente. C'est, par exemple, l'argument de Dummett contre le finitisme strict d'Esenin-Volpin ${ }^{76}$. Une grande partie de l'attrait de la logique intuitionniste vient précisément des arguments en théorie de la signification donnés par Dummett, basés sur les notions d'harmonie et de stabilité, et poursuivis par Dag Prawitz qui a vu le lien avec son principe d'inversion en déduction naturelle ${ }^{n}$. Au lieu d'une analyse de ce genre $^{78}$, Gauthier se contente de débusquer la métaphysique - celle de Thomas d'Aquin, Spinoza, etc. - qu'il voit à l'oeuvre derrière le réalisme ensembliste de Cantor. C'est, on en conviendra, insuffisant, puisque le réalisme à d'autres motivations. Il est vrai, d'autre part, qu'une telle entreprise déborde les limites de son ouvrage. Celui-ci contient cependant des paragraphes à caractère plus purement philosophique, dont ceux dédiés à Hermann Weyl et à Ludwig Wittgenstein sur lesquels je vais m'arrêter. Les écrits de ces auteurs contiennent les germes d'une philosophie de la logique que j'aurais aimé voir mise en valeur.

Mais tout d'abord, une remarque ponctuelle sur la critique concise de Frege au paragraphe 3.4 de La logique interne. Gauthier ne tient pas compte des récents travaux de Crispin Wright et George Boolos sur le système des Grundgesetze der Arithmetik, où le remplacement de l'axiome V de Frege par

76. M. A. E. Dummett, «Wang's Paradox», dans M. A. E. Dummett, Truth and other Enigmas, op. cit., p. 248-268.

77. Les te xtes principaux sont M. A. E. Dummett, « The Philosophical Basis of Intuitionistic Logic ", dans M. A. E. Dummett, Truth and other Enigmas, op. cit., p. 215-247, et D. Prawitz, « Meaning and Proofs : On the Conflict between Classical and Intuitionistic Logic », Theoria 93, 1977, p. 2-40. Je recommande tout particulièrement la lecture des chapitres 11 à 13 de M. A. E. Dummett, The Logical Basis of Metaphysics (Cambridge Mass., Harvard University Press, 1991), qui contiennent l'exposé le plus récent, qui a aussi l'avantage d'être très clair, de l'argument de Dummett. Il faut aussi mentionner les travaux de Per Martin-Löf, en particulier ses lectures à l'Université de Sienne, "On the Meaning of the Log ical Constants and the Justifications of the Logical Laws » (manuscrit, 1983), dont le contenu est très voisin.

78. Neil Tennant, pour prendre un exemple, s'est attelé à cette tâche, afin de justifier une révision en faveur d'une logique pertinente (relevant logic) intuitionniste dans Anti-Realism in Logic. Truth as Eternal (Oxford, Clarendon Press, 1987). Christopher Peacocke, quant à lui, a tenté de justifier l'interprétation réaliste des constantes logiques dans "Understanding Logical Constants" (Proceedings of the British Academy 73, 1987, p. 153-200). 
le " principe de Hume " (qui est cependant au second-ordre) rend le système consistant puisqu'il permet d'éviter la dérivation du paradoxe de ZermeloRussell $^{79}$. De plus, Gauthier qualifie les fondements logicistes frégéens de "dérivé conceptualiste de la théorie des ensembles" (p. 82). Il y a cependant tout un débat initié par Boolos, sur lequel je ne veut pas me prononcer ici, sur le point de vue de Quine selon lequel la logique du second-ordre ne serait qu'une théorie des ensembles déguisée ; l'intention de Boolos étant précisément de défendre l'autonomie de la logique du second-ordre ${ }^{80}$.

Le paragraphe dédié à Hermann Weyl (p. 87-92) reprend le texte d'une conférence donnée dans le cadre du Internationalen Hermann WeylKongresses à Kiel en 1986. La discussion de l'oeuvre de Weyl se concentre sur ses travaux en théorie des nombres et en théorie des groupes (ainsi que sur les multiples volte-faces de Weyl à l'égard de l'intuitionnisme). Malgré l'intérêt du propos, une discussion du finitisme de Weyl - en particulier de ses idées sur la quantification à l'époque de son engagement intuitionniste eût été, à mon avis, plus appropriée dans le cadre de cet ouvrage. On est réduit ici à une note en bas de page où s'est glissé, sans nul doute accidentellement, un léger contresens (p. 87, n. 18). En effet, il semble que Weyl soit le premier à avoir énoncé clairement dans son article de 1921, « Über die neue Grundlagenkrise der Mathematik ${ }^{81}$ ", la théorie du jugement selon laquelle le alle et le es gibt ne peuvent pas être lus comme une suite de conjonctions ou

79. Cf. C. I. G. Wright, Frege's Conception of Numbers as Objects, Aberdeen, Aberdeen University Press, 1983, chapitre 4, et G. Boolos, 1987, « The Consistency of Frege's Foundations of Arithmetic ", dans J. J. Thompson (dir.), On Being and Saying. Essays for Richard Cartwright, Cambridge Mass., MIT Press, 1987, p. 3-20, « Saving Frege from Contradiction», Proceedings of the Aristotelian Society 87, 1988, p. 137-151. Dans la même veine le lecteur pourra consulter R. Heck, « The Development of Arithmetic in Frege's Grundgesetze der Arithmetik ", Journal of Symbolic Logic 58, 1993, p. 579-601.

80. C'est dans Philosophy of Logic que Quine décrivait la logique comme « set theory in sheep's clothing " (Englewood Cliffs N. J., Prentice-Hall, 1971, p. 66). Les premières objections de Boolos se trouvent dans «On Second-Order Logic», Journal of Philosophy 72, 1975, p. 509-527. De George Boolos, le lecteur consultera aussi « To be is to be a value of a variable (or to be some values of some variables)», Journal of Philosophy 81, 1984 p. 430-449, « Nominalist Platonism », Philosophical Review 44, 1985, p. 327-344, et "Reading the Begriffsschrift ", Mind 94, 1985, p. 331-344.

81. H. Weyl, «Über die neue Grundlagenkrise der Mathematik», dans K. Chandrasekharan (dir.), Gesammelte Abhandlungen, Berlin, Springer, 1968, vol. 2, p. 143-180. 
de disjonctions si le domaine de quantification est infini, mais plutôt, respectivement, comme des Anweisungen auf Urteile et des Urteilsabstrakte ${ }^{82}$. Dès lors, de telles propositions arithmétiques générales ne peuvent être niées, ce qui implique que la loi du tiers-exclu ne s'applique pas à leur égard ${ }^{83}$. Cette lecture finitiste des quantificateurs a joué un rôle central dans les années 20 , puisqu'elle fut adoptée par Hilbert. Dans son fameux texte de 1927, «Über das Unendliche ", Hilbert introduit une trichotomie (et non pas simplement une dichotomie) entre énoncés "réels », énoncés " finitaires généraux " (qui correspondent à ce qu'on appelle maintenant les énoncés $-\Pi_{1}^{0}$ ) et énoncés "idéaux ». S'agissant des énoncés finitaires généraux, il adopte l'analyse de Weyl et reconnaît qu'ils ne peuvent pas être interprétés comme une infinité d'équations numériques, concaténées à l'aide des connecteurs $\wedge$ ou $\vee$. C'est à cause de cela qu'ils sont, selon lui, “incapable d'être niés ${ }^{84}$ ». En répétant Weyl presque mot pour mot, Hilbert ajoute qu'un énoncé finitaire général doit plutôt être interprété « comme jugement hypothétique qui n'asserte quelque chose que lorsqu'un numéral est donné ${ }^{85}$ ».

Il faut noter que les conceptions de Weyl ont aussi influencé Frank Ramsey, qui abandonna son point de vue ultra-platoniste en 1929, et que le parallèle avec les positions de Wittgenstein (entre 1929 et 1933) est frappant ${ }^{86}$. L'importance philosophique de ce point de vue est considérable. En effet, la position que l'on peut extraire des écrits de Weyl, Ramsey et Wittgenstein se rapproche beaucoup de l'ancien style de conventionalisme qui contestait l'interprétation sémantique de certaines classes d'énoncés - en l'occurrence ici, les énoncés mathématiques - en défendant l'idée qu'il ne s'agit que de « règles déguisées », qui n'ont pas le plein statut d'énoncés évaluables en termes de vérité et de faussetét ${ }^{87}$. Il y a là une différence fondamentale avec le

\section{Ibid., p. 155-157.}

83. Ibid, p. 158 .

84. D. Hilbert, « On the Infinite », op. cit., p. 378.

85. Ibid.

86. Sur cette question, voir M. Marion, Wittgenstein, Finitism, and the Foundations of Mathematics, à paraître, Oxford, Clarendon Press, et «Wittgenstein and Finitism », à paraître dans Synthese. À propos de l'influence de Weyl sur Ramsey, le lecteur pourra consulter U. Majer, «Ramsey's Conception of Theories : An Intuitionistic Approach ", History of Philosophy Quarterly 6, 1989, p. 233-258.

87. Dans une autre perspective, il serait opportun de comparer la distinction qui est à I'oeuvre ici à la distinction faite par Per Martin-Löf entre " propositions » et « jugements » dans « On the Meanings of the Logical Constants and the Justifications of the Logical Laws », op. cit., Intuitionistic Type Theory, Naples, Bibliopo- 
point de vue frégéen de l'anti-réalisme de Dummett qui ne rejette pas les conditions de vérité pour ces classes d'énoncés, malgré ses réserves face à la sémantique bivalente qui caractérise le réalisme. Ce point de vue est certes discrédité de nos jours. Il n'y a cependant aucune raison de croire que la victoire des frégéens soit définitive et qu'on ne puisse pas relancer ce débat.

Gauthier a considérablement adouci au cours des ans ses critiques de Wittgenstein, comme en fait foi le paragraphe qui lui est dédié. (J'ose espérer ne pas avoir été étranger à cette révision.) Il reste malgré tout encore très proche de l'interprétation faite jadis par Kreisel ${ }^{88}$, en considérant Wittgenstein comme défendant une forme de finitisme strict (p. 75, note 4) et en concluant que «Wittgenstein a produit une analyse fondationnelle de la pratique mathématique élémentaire » (p. 86). Cette conclusion est immédiatement suivie du reproche - le même que Kreisel — de ne pas s'intéresser aux mathématiques supérieures. Gauthier prend pour exemple le théorème sur la distribution des nombres premiers et il qualifie la discussion par Wittgenstein de ce théorème de "navrante à plus d'un égard " (p. 86). Reprochant à Wittgenstein de ne pas avoir utilisé les travaux de Ingham, qui était son contemporain à Cambridge, il ajoute : "Wittgenstein ne pouvait prévoir — ou l'aurait-il pu - que le théorème serait démontré constructivement " (p. 86). Cette remarque me semble injustifiée : il n'est bien sûr pas question de divination, et si Wittgenstein n'a pas « vu » qu'une preuve constructive serait un jour possible, il n'était pas plus fautif que ses contemporains, à commencer par Ingham luimême, qui disait dans son traité :

Nous pouvons en effet aller plus loin et dire qu'il est fort improbable que l'on découvre un jour une authentique preuve « à variable réelle », en tous cas tant que la théorie est fondée sur l'identité de Euler. Parce que toutes les preuves connues du théorème sur les nombres premiers sont basées sur une propriété particulière des zéros complexes de $\zeta(s)$ et, à l'inverse, ceci est une simple conséquence du théorème sur les nombres premiers lui-même. Il est donc clair que cette propriété doit être utilisée (explicitement ou implicitement) dans toute preuve qui se base sur

lis, 1984, «Truth of a Proposition, Evidence of a Judgement, Validity of a Proof », Synthese 73, 1987, p. 407-420, et « A Path from Logic to Metaphysics », dans G. Corsi \& G. Sambin, (dir.), Atti del congresso nuevi problemi della logica e della filosofia della scienza. Viareggio, 8-13 gennaio 1990, Bologne, CLUEB, vol. 2, 1991, p. 141-149. Cette distinction est à la base de sa théorie intuitionniste des types et elle s'avère très utile pour l'implémentation de celle-ci, d'où son succès auprès des informaticiens.

88. G. Kreisel, «Wittgenstein's Remarks on the Foundations of Mathematics ", British Joumal for the Philosophy of Science 9, 1958, p.135-158. 
$\zeta(s)$, et il n'est pas facile de voir comment on pourrait procéder en ne tenant compte que des valeurs réelles de $s^{89}$.

Bien sûr, Ingham s'est trompé, puisque Atle Selberg a en 1949 obtenu une preuve « élémentaire " ". Soit-dit en passant, cette preuve se distingue de la preuve classique en ce qu'elle est une preuve « directe » au sens de Kreisel, c'est-à-dire qu'elle peut être effectuée dans un système à variable libre du type proposé par Skolem sans introduire de fonctions additionelles, et non pas parce qu'elle remplace les variables complexes (de la fonction $\zeta$ de Riemann) par des variables réelles ${ }^{91}$.

J'aimerais profiter de l'occasion pour m'arrêter sur une remarque de Wittgenstein à propos du théorème sur la distribution des nombres premiers (j'oublie ici les remarques contenues dans Remarques Philosophiques, dont le manuscrit est antérieur au livre d'Ingham). Dans les Remarques sur les fondements des mathématiques, Wittgenstein ne mentionne presque jamais ce théorème, mais il fait la remarque suivante (vers 1942-44), de caractère très kantien :

L'on pourrait peut-être dire que le caractère synthétique des propositions mathématiques se manisfeste de la façon la plus évidente dans l'apparition imprévisible des nombres premiers

Mais du fait qu'ils sont soient synthétiques (en ce sens) ils n'en sont pas moins $a$ priori. J'aimerais pouvoir dire qu'on ne peut les obtenir à partir de leur concepts par un certain type d'analyse, mais plutôt qu'ils déterminent un concept par syn thèse, $[\ldots]$

La distribution des nombres premiers serait un exemple idéal de ce qu'on pourrait appeler synthétique a priori, car on peut dire qu'elle n'est en tous cas pas obtenue par une analyse du concept de nombre premier ${ }^{92}$.

89. A. E. Ingham, The Distribution of Prime Numbers, Cambridge, Cambridge University Press, 1932, p. 6. En 1938, Hardy et Wright écrivent aussi dans leur Introduction to the Theory of Numbers, où ils se limitent aux preuves élémentaires: «Nous ne pourrons pas donner une preuve, puisqu'aucune preuve élémentaire n'est connue, toutes les preuves connues dépendent de la théorie des fonctions $»(o p$. cit., p. 9)

90. Cf. A. Selberg, « An Elementary Proof of the Prime Number Theorem », Annals of Mathematics 50,1949, p. 305-313 pour la preuve originale de Selberg. Il faut noter que la preuve de Selberg (et Erdös) n'est pas entièrement satisfaisante, comme en fait foi la littérature subséquente sur l'estimation du terme correctif

91. Cf. G. Kreisel, « On the Interpretation of non-finitist Proofs. Part I », Journal of Symbolic Logic 16, 1951, p. 248.

92. L. Wittgenstein, Remarques sur les fondements des mathématiques, traduction par M.-A. Lescourret, Paris, Gallimard, 1983, p. 212. (Traduction légèrement modi- 
Lorsqu'on regarde le raisonnement qui a conduit Gauss à la conjecture :

$$
\frac{\pi(\mathbf{n})}{n} \sim \frac{1}{\log n}
$$

$(\pi(n)$ étant le nombre de nombres premiers n'excédant pas $n$, et $\sim$ le signe de l'équivalence asymptotique) on comprend le sens de la remarque de Wittgenstein. Avant Gauss, les mathématiciens ont essayé d'obtenir, sur la base des tables obtenues à partir du crible d'Ératosthène, des formules arithmétiques qui ne produraient que des nombres premiers, à défaut de tous les produire. Par exemple, Fermat avait conjecturé que tous les nombres de la forme :

$$
\mathrm{F}(n)=2^{2^{n}}+1
$$

sont des nombres premiers. Mais Euler montra que

$$
F(5)=2^{2^{5}}+1=641 \times 6700417
$$

$\mathrm{F}(5)$ n'est donc pas un nombre premier. L'intérêt des travaux de Gauss réside en partie dans l'abandon de cette approche. Il dirigea plutôt son attention sur la distribution des nombres premiers. Mais c'est encore par une étude empirique des tables de nombres premiers que Gauss trouve sa conjecture. En effet si on prend $\pi(n)$ pour le nombre de nombres premiers n'excédant pas $n$, la densité des nombres premiers pour les $n$ premiers nombres naturels est donnée par $\pi(n) / n$, et c'est en étudiant les tables de nombres premiers que Gauss s'est aperçu de l'équivalence asymptotique de $\pi(n) / n$ et de $1 / \log n$ (pour $\log n$ le logarithme népérien de $n$ ), qu'il conjectura. On voit bien ici le sens de la remarque de Wittgenstein. C'est une preuve qu'il comprenait bien le raisonnement sous-jacent à la conjecture de Gauss.

fiée.) Pour mieux comprendre le contenu kantien de cette remarque, j'aimerais citer un passage des cours sur les fondements des mathématiques de 1939 : «Une relation interne n'est jamais une relation entre deux objets, mais on pourrait dire que c'est une relation entre deux concepts. Un énoncé assertant une relation interne entre deux objets, comme par exemple un énoncé mathématique, ne décrit pas des objets mais construit des concepts » (Lectures on the Foundations of Mathematics, Ithaca, Cornell University Press, 1976, p. 73). Lorsqu'on sait le rôle joué par la notion de relation interne dans toute la philosophie de Wittgenstein, on mesure le constructivisme de Wittgenstein. Cf. A. Gargani, «Internal Relations, Syntax and Use in Wittgenstein's Philosophical Analysis ", Teoria, 1985, p. 6171 , sur le rôle de la notion de relation interne chez Wittgenstein. 
Un dernier mot sur la critique qu'adresse Gauthier à l'interprétation de Wittgenstein comme finitiste strict, par Crispin Wright ${ }^{93}$. Pour rendre justice à Wittgenstein, il ne faut pas seulement montrer qu'il n'a jamais exprimé un point de vue finitiste strict à la Esenin-Volpin, ce qui est évident ; il faut surtout montrer, contre Wright, qu'il peut aussi éviter les conclusions du finitiste strict dans ses arguments, en particulier dans sa discussion sur le caractère synoptique de la preuve ${ }^{94}$. Ici, le propos de Gauthier rejoint le mien. L'exemple choisi par Wright (qui ne tente pas de «mettre en doute» la thèse wittgensteinienne du caractère synoptique de la preuve, comme Gauthier le prétend (p. 85), mais bien d'en donner un exemple) est le théorème d'Euclide sur l'infinité des nombres premiers dont la preuve se lit comme suit : si $p_{n}$ est le plus grand nombre premier connu, on peut donc donner la liste : $p_{1} \ldots p_{n}$ à partir de laquelle on construit le nombre

$$
N=\left[p_{1} \cdot p_{2} \cdot p_{3} \ldots \cdot p_{n}\right]+1 .
$$

Ce nombre $N$ est soit premier et donc $\geq p_{n}$, ce qui contredit l'hypothèse de départ; soit il ne l'est pas et il est donc décomposable en un nombre composé et un nombre premier. Celui-ci est nécessairement $\geq p_{n}$ puisque les $p_{1} \ldots p_{n}$ doivent toujours donner un reste de 1. CQFD

Si on rejette le «non faisable » (unfeasible) comme le font les finitistes stricts, suivant en cela Esenin-Volpin, on ne peut pas donner une telle preuve puisqu'il se peut que le produit $\left[p_{1} \cdot p_{2} \cdot p_{3} \ldots \ldots p_{n}\right]$ nécessaire à la preuve ne soit pas computable pour un $p_{n}$ très grand. En d'autre termes, il semble qu'on ait ici besoin du « non faisable " pour prouver le « faisable». Wright fait la remarque suivante dans l'esprit de Wittgenstein : « on ne peut pas en général garantir l'existence d'une représentation synoptique de $n !+1$ pour un $n$ arbitraire qui soit représentable synoptiquement ${ }^{45} "$. (La formulation de Wright porte à confusion, puisque $n !+1$ n'est pas nécessaire à la preuve.) Il faut mentionner ici un résultat très inattendu de Paris, Wilkie et Woods ${ }^{96}$ qui montre que l'on a :

93. C. J. G. Wright, Wittgenstein on the Foundations of Mathematics, Londres, Duckworth, 1980.

94. Une telle interprétation est entreprise dans M. Marion, Wittgenstein, Finitism, and the Foundations of Mathematics, op. cit.

95. C. J. G. Wright, Wittgenstein on the Foundations of Mathematics, op cit, p. 138.

96. J. B. Paris, A. J. Wilkie \& A. R. Woods, « Provability of the Pigeonhole Principle and the Existence of Infinitely many Primes », Journal of Symbolic Logic 53, 1988 , p. $1235-1244$. 


\section{$\forall x \exists y>x$ ( $y$ est un nombre premier)}

dans $I \Delta_{0}+\Omega_{1}$. Ce qu'ils ont montré à la suite de Woods ${ }^{97}$, c'est que l'on peut obtenir dans $I \Delta_{0}+\Omega_{1}$ une version faible du principe combinatoire du pigeonnier :

$\Delta_{0}$-WPHP: Il n'y a pas de fonction- $\Delta_{0}$ qui soit $1-1$, telle que $f: 2 x \rightarrow x$

(à ne pas confondre avec la version dénotée dans la littérature par $\Delta_{0}$-PHP, nécessaire à la preuve de la cofinalité des nombres premiers) et que cette version suffit pour prouver l'infinité des nombres premiers (ou plutôt, pour être plus exact, le postulat de Bertrand). Il faut surtout noter ici que $\Delta_{0}$-WPHP tout comme $\Delta_{0}$ - $\mathrm{PHP}$ — n'ajoute pas de fonctions à croissance exponentielle à $\mathrm{I} \Delta_{0}+\Omega_{1}$, qui n'a donc pas besoin d'être augmenté ! Au contraire l'intérêt du résultat tient justement en ce qu'il montre qu'un principe combinatoire est suffisant, là où on pensait avoir recours à des fonctions de croissance exponentielle.

Gauthier aurait pu ou dû citer à l'appui de son propos cette preuve de l'infinité des nombres premiers dans $I \Delta_{0}+\Omega_{1}$ à l'aide du principe du pigeonnier. Grâce à ce résultat, on peut mesurer la différence entre le finitisme strict à la Esenin-Volpin, que Wright (et bien d'autres) plaque sur les écrits de Wittgenstein, et les travaux récents sur les fragments de l'arithmétique de Peano. De toute façon, une meilleure interprétation - qui va de pair avec les remarques sur l'infini, de nature aristotélicienne, que Gauthier relève (p. 83-4) - rapprochera plutôt Wittgenstein du finitisme qui est sous-jacent à certains travaux sur les fragments de l'arithmétique de Peano et sur la sous-récursion.

Gauthier objecte cependant qu'on peut « imposer une borne logarithmique à $n !+1$ de sorte que la descente devient élémentairement computable » (p. 85). Je me permets de mettre en doute cette affirmation. La fonction factorielle est exprimée dans $\mathbf{N}$ par :

97. A. Woods, "Some problems in Logic and Number Theory and their Connections », thèse de doctorat, Université de Manchester, 1981. 


$$
n !=\Pi_{p \leq n} p^{j(n, p)}
$$

avec

$$
j(n, p)=\Sigma_{i \geq 1}\left[n / p^{i}\right] .
$$

Cette fonction possède une définition $-\Delta_{0}$. Il faut noter que si $\mathbf{M}$ est un modele de I $\Delta_{0}$ dans lequel $n$ ! est totale, alors l'exponentiation est aussi totale dans ce modele. En effet, on ne peut pas dériver à partir de $2^{n}$ l'existence de $2^{n^{2}}$, dont on a besoin pour garantir l'existence de $n$ !. La fonction factorielle n'est donc pas totale dans $I \Delta_{0}+\Omega_{1}$, elle croît donc trop rapidement pour être computable en temps polynomial ${ }^{98}$. Si Gauthier propose une preuve de l'infinité des nombres premiers utilisant $n$ ! dans « A Radically Constructive Logic" ${ }^{99}$, c'est que son système n'est pas aussi faible qu'il veut bien le croire.

\section{Conclusion}

Je me suis contenté pour l'essentiel d'indiquer que si Gauthier insiste pour proposer un constructivisme radical, il aurait avantage à adopter un formalisme plus restrictif que celui de son arithmétique de Fermat. Selon moi, l'arithmétique bornée, dont l'intérêt fondationnel a été mis en valeur par Edward Nelson, présente un cadre formel plus faible que le sien. Cependant, je ne cache pas que le programme modifié de Hilbert soit sans faille et il n'est pas sûr non plus que le point de vue $\mathrm{I} \Delta_{0}+\Omega_{1}$ corresponde à une position philosophique cohérente. D'autre part, il me semble que certains aspects des positions philosophiques de Gauthier le poussent vers des positions finitistes strictes et il gagnerait à examiner celles-ci de plus près. Je pense en particulier à la notion de prédicativité.

Il me faut, en terminant, reconnaître l'acuité et l'originalité de la vision philosophique d'Yvon Gauthier, qui n'a que peu ou point d'équivalent dans le monde francophone. Gauthier a en effet le mérite d'avoir développé au cours des ans des idées qui lui sont propres, ce que peu d'auteurs peuvent revendiquer. Quoi que l'on dise de l'arithmétique de Fermat, il reste que les questions

98. Sur ce sujet, voir P. D’ Aquino, «Exponentiation and Fragments of Arithmetic », thèse de doctorat, Université d'Oxford, 1992, et «Local Behaviour of the Chebyshev Theorem in Models of $\mathrm{I} \Delta_{0} \ldots$, op. cit.

99. Y. Gauthier, "A Radically Constructive Logic», Cahiers du département de philosophie, Université de Montréal, 92-06, 1992. 
soulevées par les travaux de Gauthier sont parmi les plus fondamentales qui soient en philosophie des mathématiques ${ }^{100}$.

Departement de philosophie

Université d'Ottawa

100. J'aimerais remercier Gianluigi Bellin, Roy Dyckhoff, Daniel Isaacson, Georg Kreisel, Hughes Poltier et tout particulièrement Angus Macintyre pour leur aide apportée à la rédaction de cette étude, entreprise lorsque l'auteur était rattaché successivement à l'Université de St. Andrews et à l'Université de Boston, ces séjours ayant été rendus possibles grâce à l'octroi d'une bourse de recherche postdoctorale du Conseil pour la Recherche en Sciences Humaines du Canada. Je dois aussi remercier Yvon Gauthier, pour ses commentaires sur une version préliminaire de cette étude. 